\title{
Optimal parameterization of tire-rim interaction for aircraft wheels
}

\author{
K. Cosseron* \\ Laboratoire de Mécanique et Technologie (LMT), ENS Paris-Saclay, CNRS, Univ. Paris-Saclay, 94235 Cachan, France \\ Safran Group, Safran Landing Systems, 78140 Vélizy-Villacoublay, France \\ D. Mellét \\ Safran Group, Safran Landing Systems, 78140 Vélizy-Villacoublay, France \\ now at Safran Group, Safran Tech, 78772 Magny-les-Hameaux, France \\ F. Hild ${ }^{\ddagger}$ and S. Roux ${ }^{\S}$ \\ Laboratoire de Mécanique et Technologie (LMT), ENS Paris-Saclay, CNRS, Univ. Paris-Saclay, 94235 Cachan, France
}

The knowledge of tire-rim interface loadings is crucial to design aircraft wheels. A parameterization of these loadings is proposed using model reduction techniques. The static eigenmodes of the wheel stiffness matrix that is condensed at the tire-rim interface provide a suited hierarchy of displacement or loading modes that can be sorted according to their elastic energy. A truncation of this series at a chosen order is performed to approximate any load applied to the rim. The use of cyclic symmetry is shown to lead to an efficient numerical scheme to compute these modes. This parameterization does not involve the tire modeling, which is beyond the control of aircraft wheel manufacturers. The reduced basis composed of the major eigenmodes is shown to be more compact and robust than other parameterizations based on arbitrary shape functions or tire models and will be further used for inverse identifications of tire-rim loadings. Moreover, this approach is generic and offers a convenient and efficient framework for arbitrary loadings.

\footnotetext{
*Ph.D. Student, Mechanics and Materials Division. Corresponding author. Email: cosseron@1mt.ens-cachan.fr.

${ }^{\dagger}$ Mechanical and Materials Engineering Expert, Materials and Processes Division.

${ }^{\ddagger}$ Research Professor, Mechanics and Materials Division.

${ }^{\S}$ Research Professor, Mechanics and Materials Division.
} 


\section{Nomenclature}

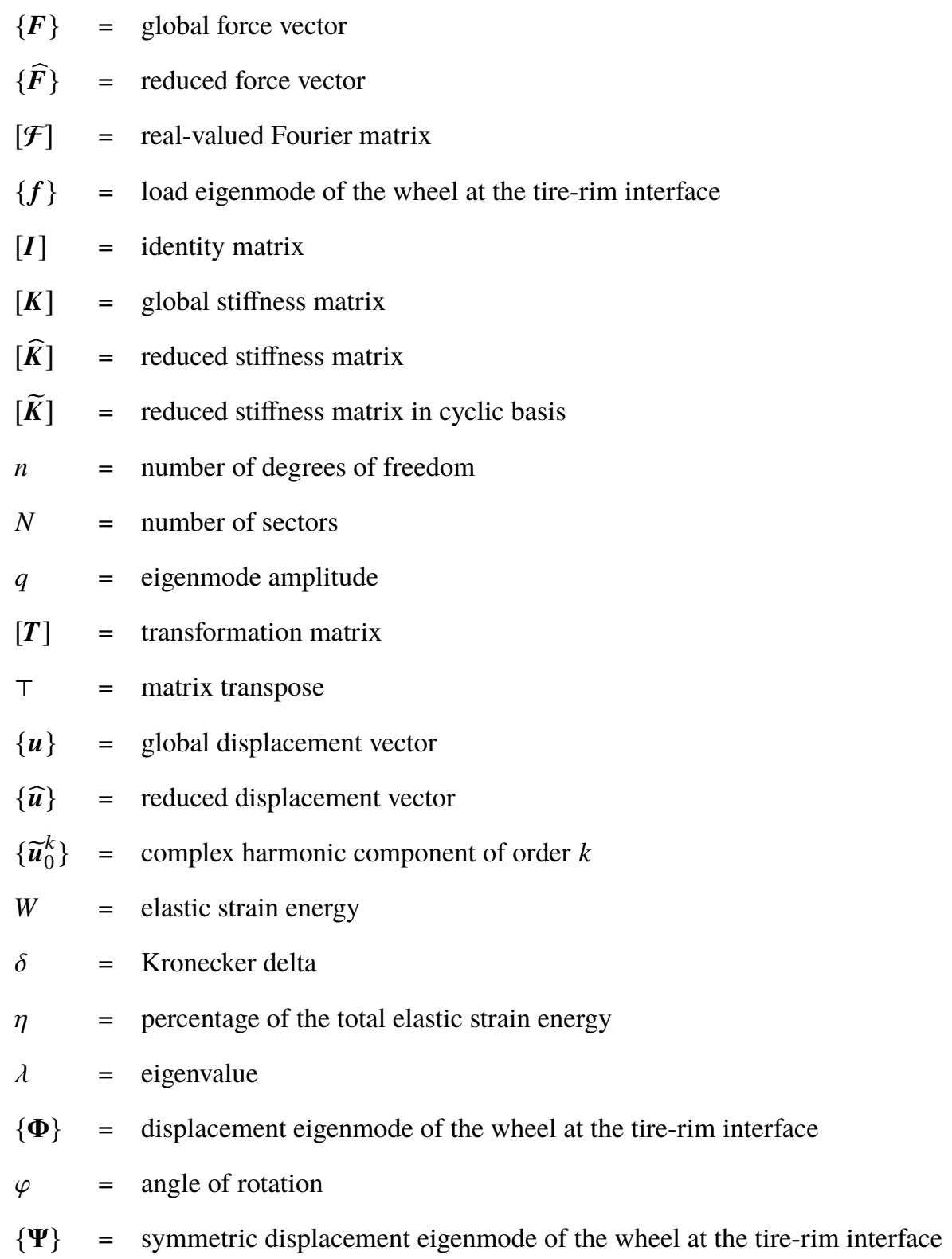




\section{Introduction}

HE predictive character of numerical simulations run to design a structure critically depends on the accuracy with
which the boundary conditions prescribed on the structure are introduced [1--8]. For aircraft wheel manufacturers, the loadings applied to the wheel during ground maneuvering by various tires that are mounted play an important role. However, they are hardly known apart from the resultant force between the ground and the tire. Aircraft wheel manufacturers do not control the tire, which is a complex intermediate component, but must still work with it to design their products. Because tire-ground loads are transmitted to the wheel through the tire at the tire-rim interface, assumptions on the deformation behavior of the tire are to be made to determine the correct applied loading to the wheel and many experiments are often required to evaluate the wheel structural response [9, 10].

Various attempts have been performed to experimentally investigate tire-rim interface loadings. Zaharov [11], Balabin et al. [12], and Walter et al. [13] used strain gauges and magnetostrictive transducers to directly measure normal contact pressure distributions at the tire-rim interface for automotive wheels. These measurement techniques required some operations (i.e., welding or drilling) to mount the sensors on the wheel rim. Therefore, they were very intrusive and could modify the tire-rim interface behavior. Sherwood et al. [14] used flexible and thin piezoelectric films to minimize the intrusion in the tire-rim contact area. With new technologies now available, Wan et al. [15] also recorded the interface pressure distribution with the Tekscan's I-Scan System. However, all of these instrumentations generally lead to inaccurate results due to their delicate calibration. The shape of the measured pressure distribution is fairly reliable whereas the accuracy of its amplitude is highly dependent on the performed calibration. Moreover, these measurement techniques are fragile and can be damaged because of the shear forces encountered in the contact area during tire-wheel assembly and loading cases.

Other investigations moved toward measurements outside the tire-rim contact area to identify loadings using inverse methods. Sherwood et al. [14, 16] performed holographic interferometry measurements to obtain a map of the out-of-plane displacements on the inboard and outboard halves of an aircraft wheel during an inflation case. A three-dimensional (3D) finite element analysis was then conducted to back-calculate the tire-rim contact pressure distribution based on these experimental data and the results were compared with those obtained with piezoelectric films. Both methods showed similar trends in the overall contact pressure variations qualitatively, but not quantitatively.

Spencer et al. [17], Kirkner et al. [18], Schudt et al. [19], and Kandarpa et al. [20] proposed a combined analyticalexperimental methodology that recovers the tire-rim interface pressure distribution with a given number of experimental strain measurements on the wheel. The loading was described as a double Fourier series and the components were determined by least squares fit using the experimentally measured strains. The methodology was applied for inflation and vertical loading cases. The influence of the number and location of strain gauges was also studied. However, the focus of these papers was the ability of the algorithm to recover a given pressure distribution rather than assessing the required accuracy on tire-rim loadings to obtain a reasonable approximation of the wheel structural response. 
Lastly, some authors dealt with the modeling of the complete tire-rim assembly. Chen et al. [21] made an attempt to theoretically predict the bead area contact load at the tire-rim interface assuming the wheel to be completely rigid. Along the same lines, Tseng et al. [22] studied the effect of the interference fit between the tire bead and the rim. More recently, Meng et al. [23] proposed simplified functions to approximate contact pressure distributions on the bead and flange areas for an automotive wheel, which were based on the results of a finite element model of the tire-wheel assembly. Furthermore, Ballo et al. [24] proposed a semi-analytical tire model to compute the reaction forces acting on the tire-rim interface. These studies are highly dependent on the material properties used for the tire model and almost always consider a totally rigid wheel.

The knowledge of tire-rim interface loadings would provide an invaluable help to shorten aircraft wheel design processes. To tackle this challenge, the authors have proposed an inverse identification procedure of tire-rim loadings [25, 26] for several cases (i.e., inflation, vertical and cornering). The aim is to identify the loadings applied by a tire on an aircraft wheel via the measurement of the wheel deformation (e.g., displacement fields obtained by Digital Image Correlation analyses, strains at gauge locations). The underlying tasks are to define an objective parameterization of tire-rim loadings, which is as robust and compact as possible, and to choose appropriate measurements to be able to calibrate the loading parameters. A Finite Element Model Updating (FEMU) algorithm will then be used to solve this inverse problem. This paper focuses on the parameterization of tire-rim interface loadings for which several proposals were made in the litterature. Some authors used Fourier series [17--20] or cosine functions [27], which do not involve any specific knowledge on the mechanical behavior of the tire-rim interface, while others defined simplified functions based on tire models [23, 24]. In this paper, a paradigm change from the current litterature is introduced. It is proposed to define such a parameterization using the static eigenmodes of the wheel at the tire-rim interface, which are only dependent on the wheel structure and do not explicitly involve tire modeling.

The paper is organized as follows. Section $\Pi$ is devoted to the presentation of the methodology for the determination of the tire-rim interface static eigenmodes. Model reduction techniques and properties of structures with cyclic symmetry are used. Section [II] discusses criteria to determine how many modes must be retained to provide a good approximation of a given static loading. The criteria proposed herein are the conservation of the total elastic strain energy and the convergence of other macroscopic quantities characterizing the loading case such as the resultant forces and average displacements in the tire-rim contact area. In Section [V] the proposed parameterization is assessed by projecting known tire-rim loadings on the eigenmode basis. A comparison between known and approximate tire-rim interface loadings is presented. The results are intended for use in future studies of the impact of approximate loadings on the wheel structural response. 


\section{Static eigenmodes of the wheel at the tire-rim interface}

This section is devoted to the methodology for the determination of the static eigenmodes of the wheel at the tire-rim interface, which will serve as the basis to approximate any load applied on the rim. First, the general approach is detailed and involves model reduction techniques. Then, properties of structures with cyclic symmetry are used to make the procedure more effective, requiring the modeling of one fundamental sector of the wheel only. Finally, the obtained static eigenmodes of the wheel at the tire-rim interface are presented.

\section{A. General methodology}

A 3D finite element model of an aircraft wheel is considered (Fig. 1). It contains two wheel halves (inboard and outboard) and tie bolts to join them. The model involves multiple nonlinear contacts and the material properties are those of an aluminum alloy with a linear elastic behavior for the wheel and steel for other parts.

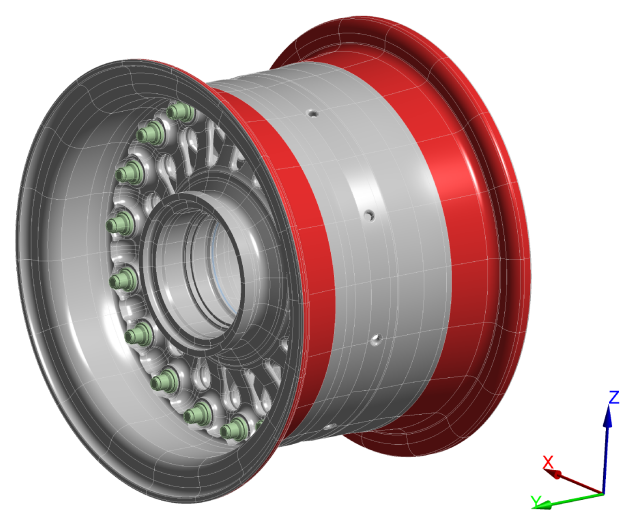

Fig. 1 Finite element model of an aircraft wheel

The finite element model is characterized by its stiffness matrix $[\boldsymbol{K}]$, which describes the relationship between the nodal forces gathered in the column vector $\{\boldsymbol{F}\}$ and the nodal displacement vector $\{\boldsymbol{u}\}$

$$
\{\boldsymbol{F}\}=[\boldsymbol{K}]\{\boldsymbol{u}\}
$$

The tire-rim interface area, which is highlighted in red in Fig. 1, is defined by the Aircraft Year Book from the Tire and Rim Association [28]. It corresponds to the widest tire-rim contact area and ensures the compatibility of both components. Thus, tire-rim interface loadings, except nitrogen pressure that is known to be uniform, only occur on this area. To define a parameterization of these loadings, a static condensation [29] of this model is performed on the tire-rim interface degrees of freedom (DOFs). This condensation consists in separating the DOFs of the whole structure into master and slave DOFs, which are denoted with $m$ and $s$ subscripts respectively 


$$
\left\{\begin{array}{c}
\left\{\boldsymbol{F}_{s}\right\} \\
\left\{\boldsymbol{F}_{m}\right\}
\end{array}\right\}=\left[\begin{array}{ll}
{\left[\boldsymbol{K}_{s s}\right]} & {\left[\boldsymbol{K}_{s m}\right]} \\
{\left[\boldsymbol{K}_{m s}\right]} & {\left[\boldsymbol{K}_{m m}\right]}
\end{array}\right]\left\{\begin{array}{c}
\left\{\boldsymbol{u}_{s}\right\} \\
\left\{\boldsymbol{u}_{m}\right\}
\end{array}\right\}
$$

Therefore, the DOFs of the tire-rim interface are chosen hereafter as master DOFs. Static condensation also assumes that the forcing terms on the slave DOFs are vanishing. A reduction of the initial mechanical problem from Eq. (1) is then obtained

$$
\{\widehat{\boldsymbol{F}}\}=[\widehat{\boldsymbol{K}}]\{\widehat{\boldsymbol{u}}\}
$$

with $[\widehat{\boldsymbol{K}}]=[\boldsymbol{T}]^{\top}[\boldsymbol{K}][\boldsymbol{T}],[\boldsymbol{T}]^{\top}=\left[-\left[\boldsymbol{K}_{m s}\right]\left[\boldsymbol{K}_{s s}^{-1}\right] \quad[\boldsymbol{I}]\right],\{\widehat{\boldsymbol{F}}\}=[\boldsymbol{T}]^{\top}\{\boldsymbol{F}\}$, and $\{\widehat{\boldsymbol{u}}\}=\left\{\boldsymbol{u}_{m}\right\}$. The reduced problem from Eq. (3) gives the force-displacement relationship for the tire-rim interface nodes, with no loss of accuracy because all elements of the original stiffness matrix contribute. However, one can notice that this reduction technique implies the numerical model to be linear, and all nonlinear contacts are therefore linearized during this process. The underlying assumption is that the two wheel halves cannot be separated, which is not far from reality because of the large amount of bolt tightening. Thus, it is assumed that this linearization has a minor impact on the tire-rim interface area behavior (i.e., Saint-Venant's principle). Then, the eigenvalues, $\lambda_{i}$, and eigenmodes, $\left\{\boldsymbol{\Phi}_{i}\right\}$, of $[\widehat{\boldsymbol{K}}]$ are sought

$$
\left([\widehat{\boldsymbol{K}}]-\lambda_{i}[\boldsymbol{I}]\right)\left\{\boldsymbol{\Phi}_{i}\right\}=\{\boldsymbol{0}\}
$$

where the index $i$ is conventionally chosen so that the eigenvalues are sorted in increasing order. The symmetry of the stiffness matrix and the normalization of the eigenvectors to a unit norm implies that the eigenvectors form an orthonormal basis

$$
\left\{\boldsymbol{\Phi}_{i}\right\}^{\top}\left\{\boldsymbol{\Phi}_{j}\right\}=\delta_{i j}
$$

These eigenmodes, which are orthogonal to each other, are called the static eigenmodes of the wheel at the tirerim interface. They constitute a complete set of generalized shape functions for describing the tire-rim interface displacements [30]. Thus, for a given tire-rim interface loading $\{\widehat{\boldsymbol{F}}\}$, the associated displacements $\{\widehat{\boldsymbol{u}}\}$ are expressed as a linear combination of these eigenmodes

$$
\{\widehat{\boldsymbol{u}}\}=\sum_{i=1}^{n} q_{i}\left\{\boldsymbol{\Phi}_{i}\right\}
$$

where $n$ is the number of DOFs of the tire-rim interface. The undetermined amplitudes $q_{i}$ are found by pre-multiplying Eq. (3) by the transpose of each eigenvector and using the orthogonality property from Eq. (5). For the $k$-th eigenmode, one obtains

$$
q_{k}=\left\{\boldsymbol{\Phi}_{k}\right\}^{\top}\{\widehat{\boldsymbol{u}}\}=\frac{1}{\lambda_{k}}\left\{\boldsymbol{\Phi}_{k}\right\}^{\top}\{\widehat{\boldsymbol{F}}\}
$$


The static eigenmodes of the wheel at the tire-rim interface can also be considered as eigenload cases $\left\{\boldsymbol{f}_{k}\right\}$ when they are pre-multiplied by the reduced stiffness matrix or by their associated eigenvalue

$$
\left\{\boldsymbol{f}_{k}\right\}=[\widehat{\boldsymbol{K}}]\left\{\boldsymbol{\Phi}_{k}\right\}=\lambda_{k}\left\{\boldsymbol{\Phi}_{k}\right\}
$$

The eigenvalue $\lambda_{k}$ is interpreted as the wheel stiffness for the eigenload case $\left\{\boldsymbol{f}_{k}\right\}$. By extension, any load $\{\widehat{\boldsymbol{F}}\}$ applied to the rim is expressed as a linear combination of the eigenloads

$$
\{\widehat{\boldsymbol{F}}\}=\sum_{i=1}^{n} q_{i}\left\{\boldsymbol{f}_{i}\right\}=\sum_{i=1}^{n} q_{i} \lambda_{i}\left\{\boldsymbol{\Phi}_{i}\right\}
$$

\section{B. Use of cyclic symmetry properties}

The reduced stiffness matrix $[\widehat{\boldsymbol{K}}]$, which is required to determine the static eigenmodes of the wheel at the tire-rim interface, can be manually obtained by successively prescribing unitary displacements to tire-rim interface DOFs while setting all others to zero. The number of calculations is thus equal to the number of tire-rim interface DOFs. However, the finite element software can generate reduced matrices (i.e., superelements) in an automated way [31]. The finite element model depicting a 360-degree wheel is computationally expensive and the number of tire-rim interface DOFs is large (i.e., several tens of thousands). Although the static condensation allows the stiffness matrix dimension to be reduced, the sparsity of the original stiffness matrix is lost and the reduced stiffness matrix is dense, which can lead to storage issues. Thus, there is a need to obtain and diagonalize the reduced stiffness matrix in a more compact way.

Due to their geometrical properties, aircraft wheels belong to the class of periodic structures with cyclic symmetry. They are defined by a circular and closed replication about an axis of symmetry of a minimal entity called a sector. The studied wheel is composed of $N=9$ identical sectors, with an angle $\alpha=2 \pi / N$ between them, numbered from 0 to $N-1$, as shown in Fig. 2. A fundamental sector of the wheel (green) contains one generation sector (blue) and its mirror (orange). This additional symmetry property defines a dihedral symmetry [32]. The following paragraph briefly describes the methodology to obtain the desired reduced stiffness matrix using properties of structures with cyclic symmetry and thus modeling only one fundamental sector of the wheel. A detailed review of the equations behind this methodology, which is illustrated in Fig. 3, is given in the Appendix. 


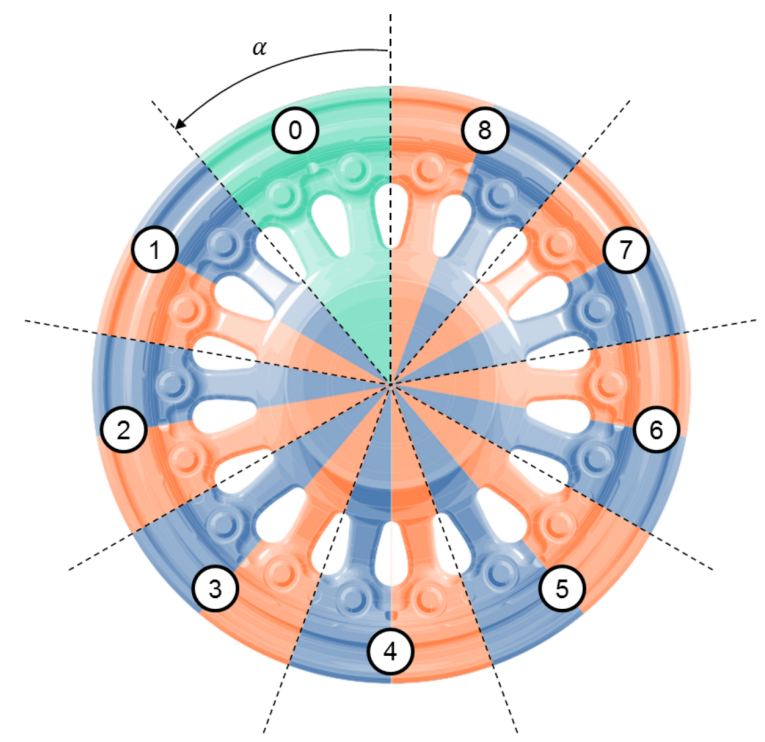

Fig. 2 Cyclic symmetry of an aircraft wheel

Let us consider a fundamental sector of the aircraft wheel, as shown in green in Fig. 2. First, a static condensation of this sector on its inter-sector boundaries and tire-rim interface DOFs is performed, thereby leading to a reduced stiffness matrix $\left[\widehat{\boldsymbol{K}}_{\text {sec }}^{\text {bound }+i n t}\right]$. Then, cyclic symmetry properties are used to build the reduced stiffness matrix of the wheel at all of its inter-sector boundaries and at the tire-rim interface $\left[\widehat{\boldsymbol{K}}_{360}^{\text {bound }+i n t}\right]$. Last, a second static condensation is performed to obtain the reduced stiffness matrix of the wheel at tire-rim interface DOFs only. This matrix, denoted as $\left[\widehat{\boldsymbol{K}}_{360}^{\text {int }}\right]$, is block-circulant and all its sub-blocks are known at this point. A coordinate transformation (i.e., from physical to cyclic components) gives a pseudo-block-diagonal matrix in cyclic basis. Therefore, the diagonalization of $\left[\widehat{\boldsymbol{K}}_{360}^{\text {int }}\right]$ required to obtain the tire-rim interface static eigenmodes is split into several smaller sub-problems, defined in the cyclic basis and depending upon a harmonic index $k$, which are easier to address. Solutions to these eigenproblems are back-transformed into the physical basis, and reveal non-degenerated and degenerated solutions characterized by the existence of nodal diameters in mode shapes. 


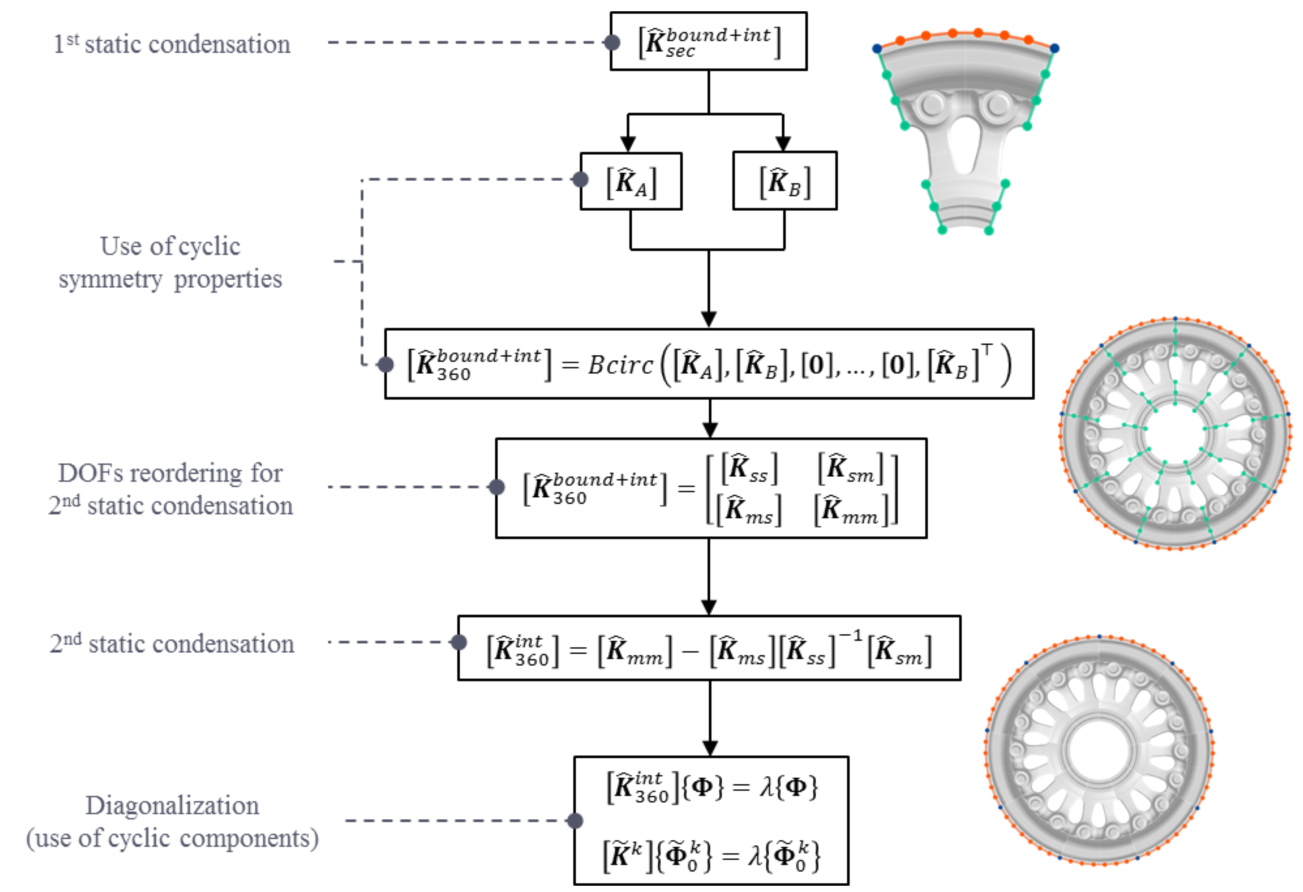

Fig. 3 Framework for tire-rim interface static eigenmodes determination using cylic symmetry properties

\section{Results}

The finite element model of the fundamental sector used to obtain the static eigenmodes of the wheel at the tire-rim interface is illustrated in Fig. 4 . The mesh consists of approximately 100,000 elements and 30,000 nodes (including almost 2,000 nodes for the tire-rim interface area). Linear elements are used to limit the number of DOFs, and thus the stiffness matrix size. A side study showed that low-frequency eigenmodes are not very sensitive to the mesh size. Boundary conditions are a fixed support on bearing support surfaces and cyclic symmetry condition on sector boundaries.

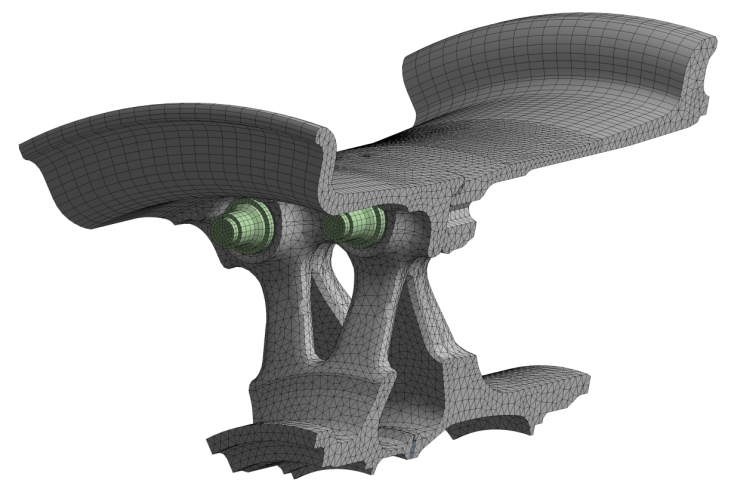

Fig. 4 Fundamental sector mesh 
Applying the methodology described in Section II.B, the static eigenmodes of the wheel are obtained at the tire-rim interface, ordered by increasing eigenvalue for each harmonic index. For eigenmodes of harmonic 0 , all sectors of the wheel are in phase (Figs. 5,6). Moreover, displacements with high dynamic ranges are essentially axisymmetric for low-frequency modes. Thus, for the study of an inflation case for example, only modes of this harmonic order will be considered.

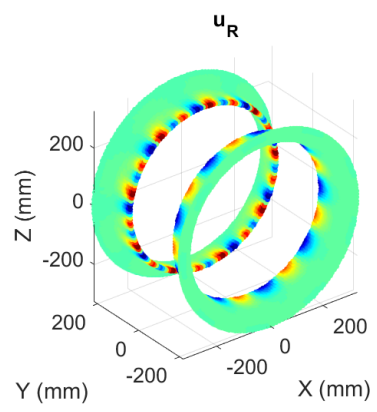

(a) Radial displacement

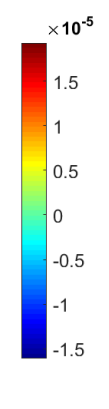

(b)

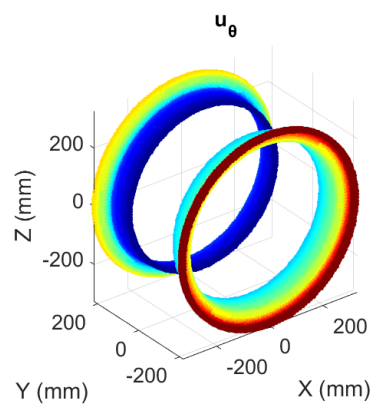

(b) Circumferential displacement

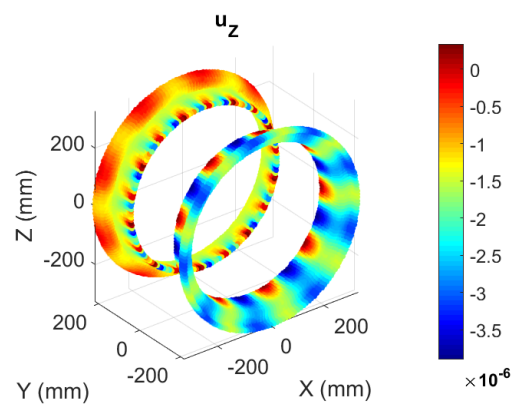

(c) Axial displacement

Fig. 5 First mode of harmonic 0 . Note that $u_{R}$ scale is of order $10^{-5}$, and that of $u_{Z}$ is $10^{-6}$, much smaller than $u_{\theta}$ that is of order $10^{-2} \mathbf{m m}$

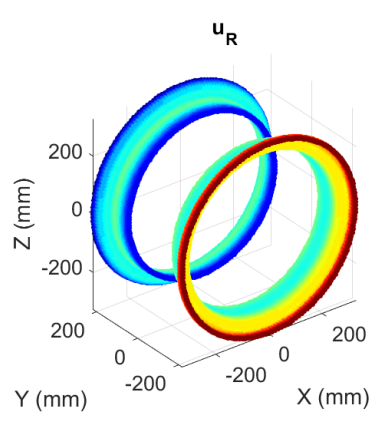

(a) Radial displacement

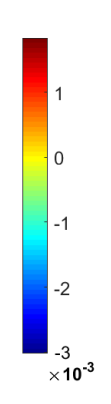

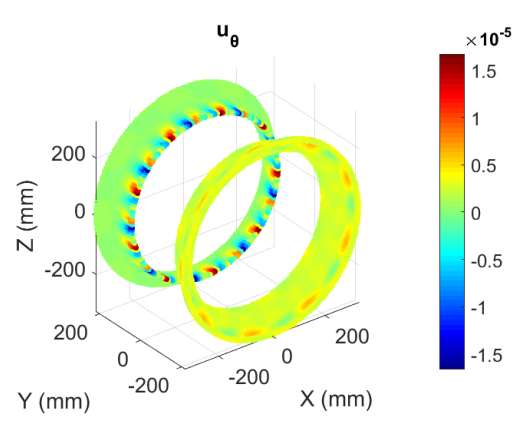

(b) Circumferential displacement

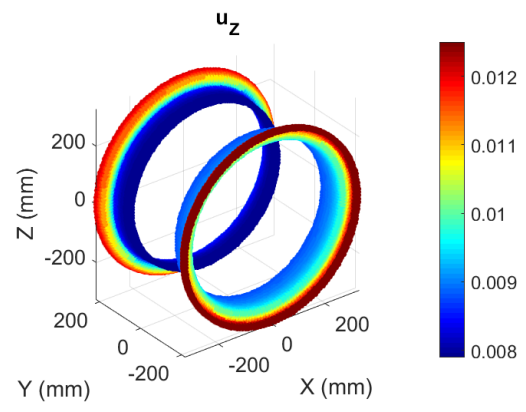

(c) Axial displacement

Fig. 6 Second mode of harmonic 0 . Note that $u_{R}, u_{\theta}, u_{Z}$ are respectively of order $10^{-3}, 10^{-5}$ and $10^{-2} \mathrm{~mm}$

Then, when the harmonic index increases $(k \in[1, K])$, nodal diameters appear in the mode shapes, generating an inter-sector phase shift. For eigenmodes of harmonic 1, Figs. 7 and 8 exhibit one nodal diameter. These figures also show the two modes of a single pair, orthogonal to each other and associated with the same eigenvalue. 


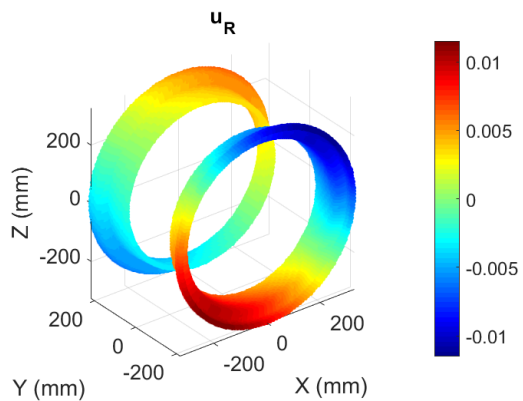

(a) Radial displacement

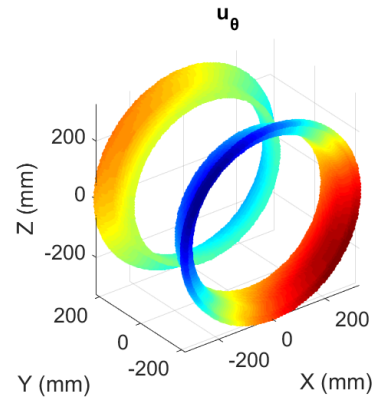

(b) Circumferential displacement

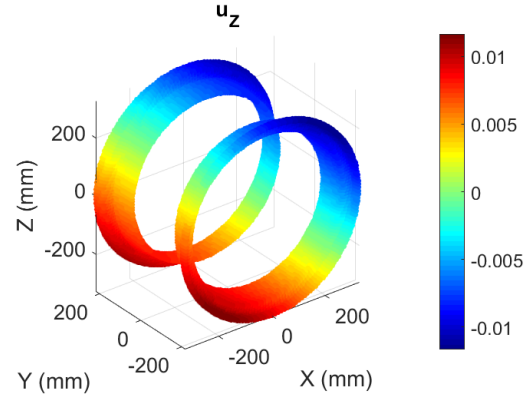

(c) Axial displacement

Fig. 7 First mode of harmonic 1

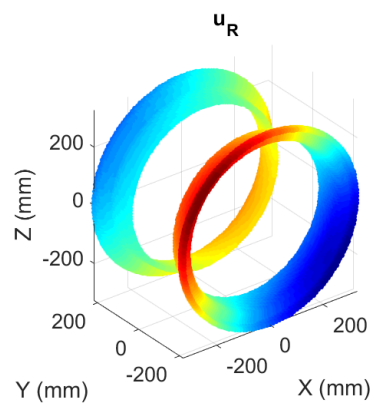

(a) Radial displacement

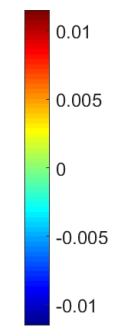

(1)

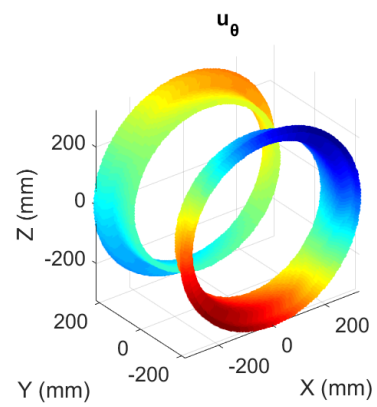

(b) Circumferential displacement

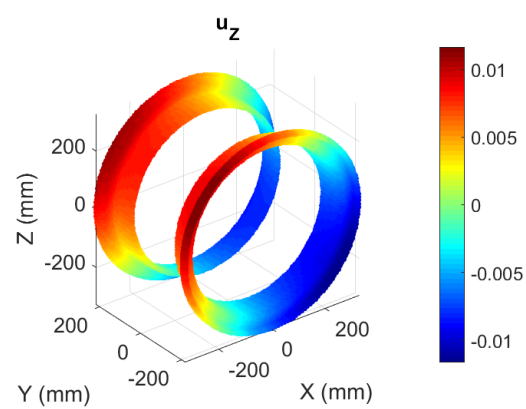

(c) Axial displacement

Fig. 8 Second mode of harmonic 1

Because any linear combination of these two complementary modes is also a static eigenmode of the wheel at the tire-rim interface, this property can be used to generate a new eigenmode basis that meets certain conditions. Aircraft wheel manufacturers generally assume a symmetric behavior of static tire-rim loadings along the $Y-Z$ plane. Therefore, considering eigenmodes $\left\{\boldsymbol{\Phi}_{i}\right\}$ and $\left\{\boldsymbol{\Phi}_{i+1}\right\}$ of a single pair, a symmetric eigenmode $\left\{\boldsymbol{\Psi}_{i}\right\}$ is generated

$$
\left\{\boldsymbol{\Psi}_{i}\right\}=\left\{\boldsymbol{\Phi}_{i}\right\} \cos (\varphi)+\left\{\boldsymbol{\Phi}_{i+1}\right\} \sin (\varphi)
$$

determining the appropriate value of angle $\varphi$. Hence, the number of eigenmodes for each harmonic index $k \in[1, K]$ is divided by two and equal to the number of eigenmodes for harmonic 0 . An example of a symmetric eigenmode, generated using the first two modes of harmonic 1, is shown in Fig.9 


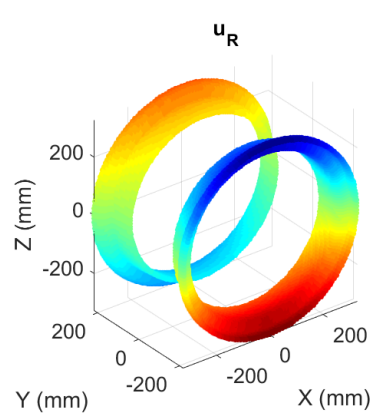

(a) Radial displacement

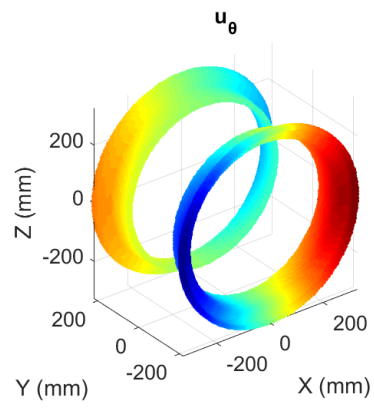

(b) Circumferential displacement

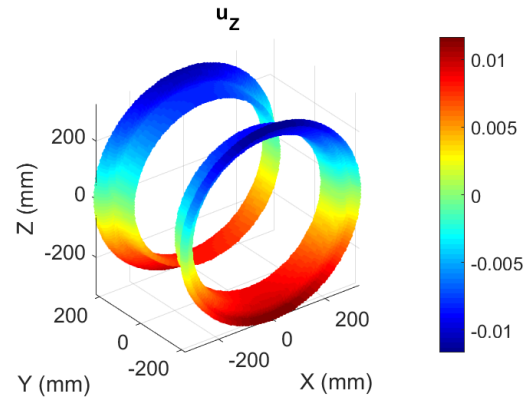

(c) Axial displacement

Fig. 9 First symmetric mode of harmonic 1

In the remainder of this paper, only symmetric eigenmodes are considered for harmonic index $k \in[1, K]$. However, eigenmodes will still be denoted with the $\{\boldsymbol{\Phi}\}$ symbol for the sake of simplicity.

\section{Selection criteria}

The parameterization proposed in Section $[\Pi$ gives an objective description of the tire-rim loadings from the point of view of an aircraft wheel manufacturer. The eigenmode basis retains the initial number of tire-rim interface degrees of freedom and only corresponds to a different representation of the tire-rim loadings (i.e., basis change) with no particular assumptions. The mathematical problem is thus strictly equivalent but easier to handle because of the diagonalization of the wheel stiffness matrix that is condensed at the tire-rim interface. This parameterization is intended for use in future inverse identifications of tire-rim loadings. Therefore, the number of loading parameters to be identified is crucial and should be minimized. In this section, selection criteria are suggested to reduce the number of selected modes to a minimum during the approximation of a given static loading with the least consequences in terms of global elastic energy. In this sense, the chosen basis is optimal.

\section{A. Elastic strain energy conservation}

In modal analyses, vibration modes are used to approximate the dynamic response of structures during excitations. The common practice is to consider eigenmodes associated with eigenvalues within a particular range, depending on the excitation frequency [33]. However, in static analyses, there is no reason for these selected eigenmodes to effectively approximate the displacements induced by a given static loading. Thus, the frequency criterion does not make sense and will not be used.

To reveal dominant modes, as proposed by Sobieszczanski et al. [30], a criterion based on the elastic strain energy conservation is chosen. For a given static loading $\{\widehat{\boldsymbol{F}}\}$ on the tire-rim interface, the total elastic strain energy $W_{\text {tot }}$ is computed as

$$
W_{\text {tot }}=\frac{1}{2}\{\widehat{\boldsymbol{u}}\}^{\top}[\widehat{\boldsymbol{K}}]\{\widehat{\boldsymbol{u}}\}
$$


Projecting this static loading on the tire-rim interface static eigenmode basis, the elastic strain energy contribution of the $k$-th eigenmode is obtained using Eqs. (4) to (6), and (11)

$$
W_{k}=\frac{1}{2} \lambda_{k} q_{k}^{2}=\frac{1}{2 \lambda_{k}}\left(\left\{\boldsymbol{\Phi}_{k}\right\}^{\top}\{\widehat{\boldsymbol{F}}\}\right)^{2}
$$

Thus, sorting the modes by descending magnitude of the associated elastic strain energy, the modes that strongly contribute to the approximation of the given static loading can be selected. The following criterion is then defined to determine the number of eigenmodes to be selected

$$
W=\sum_{i} W_{i} \geq \eta \times W_{t o t}
$$

where $\eta$ is expressed as a percentage of the total elastic strain energy. Note that a truncation of the series of modes to a given order means implicitly that higher order modes amplitudes are forced to 0 . Thus, this truncation can be seen as a sparsity requirement (because the truncation order is generally much smaller than the total number of degrees of freedom of the tire-rim interface). Moreover this sparsity is designed to optimally preserve the energy of the system [34]. Only modes that significantly contribute to the total elastic strain energy are selected. This criterion is consistent with future inverse identifications of the tire-rim loadings which will be performed because it can be directly related to the structural impact induced in the elastic regime. In other words, it allows eigenmodes associated with a low elastic strain energy, which have a minor impact on the structural response of the wheel and hence whose identifiability will be poor (i.e., characterized by a high uncertainty), to be ignored. The optimality of the proposed parameterization is thus claimed in a specific direction, namely defined by the elastic strain energy, and not universally. The criterion cannot comprehensively ensure the convergence of various quantities of interest observed in different areas of the wheel. It corresponds to a particular analysis viewpoint, which is characterized by its own metric, and suited to the problem of inverse identification of tire-rim loadings. Other viewpoints may be envisioned. For example, if a particular quantity of interest is considered from a design perspective (e.g., Von Mises stress at critical points of the wheel), a new criterion would be defined to select the relevant modes accordingly.

\section{B. Additional criteria}

The elastic strain energy conservation criterion allows the number of selected modes to be drastically reduced. However, this is a macroscopic criterion. To assess the accuracy of the approximation, it is necessary to define other quantities of interest, and to monitor their convergence with the number of selected modes.

First, the resultant forces are considered. Because tire-ground loads are known by aircraft wheel manufacturers, the approximate loading must maintain them to be valid. Resultant forces $F_{X}, F_{Y}$, and $F_{Z}$ in the three directions of the 
Cartesian coordinate system on the inner (IWH) and outer (OWH) wheel halves are monitored. It is noteworthy, however, that the resultant forces are naturally associated with low-order modes, while high-order ones are naturally obtained for locally self-balanced loads so that they only affect a small confined region or boundary layer. Thus, although this second criterion of resultant forces seems not to be in line with the previous one based on modes, in fact they are close by construction. This question can be documented numerically by considering (as a function of $k$ ) the corresponding $\left\{\boldsymbol{f}_{k}\right\}$ modes attached to each resultant.

Then, the approximated displacements in the tire-rim interface area can be compared to the real ones to get more local information. Some modes have a minor contribution to the total elastic strain energy or resultant forces. However, they can significantly modify the local tire-rim loading distribution and thus affect the structural response of the wheel. Last, one can move outside the tire-rim interface area and look at the impact of the approximate loading on critical areas of wheel. For example, local strains in the rim and webs can be investigated to assess the sensitivity of these areas to the tire-rim loading. The main aim of the approximation is then to ensure an accurate representation of the structural response rather than recovering exactly a given tire-rim loading. Assessing the impact of the approximate loading on critical areas of the wheel requires the use of the full finite element model of the wheel and will be investigated in future studies. Nonetheless, because the assembly area of the two wheel halves acts like a low-pass filter, the quantities of interest below this area will thus be well predicted once the correct resultant forces are introduced in the finite element model. In an obvious manner, a larger number of modes will be required to predict quantities of interest located near the tire-rim contact area (i.e., Saint-Venant's principle).

\section{Projection of known tire-rim loadings}

In this section, known tire-rim loadings given by tire manufacturers are projected onto the eigenmodes basis defined in Section $\Pi$ A. The selection criteria suggested in Section $\amalg$ are then used to determine the number of modes required to effectively approximate these loadings.

\section{A. Analyzed data}

Tire manufacturers usually give maps of the contact pressure distribution at the tire-rim interface for different loading cases to support aircraft wheel manufacturers in the development of their products. These data are used to evaluate the structural response of the wheel before performing validation tests and are thus very useful for the aircraft wheel designer. Two radial tires (designated hereafter as A and B) that can be mounted on the studied wheel are investigated. Table 1 describes loading cases for which tire-rim contact pressure distributions are available. These data are obtained by tire manufacturers using finite element analyses for tire A, and experiments for tire B. However, the wheel is considered totally rigid in both cases, which is a strong assumption for the aircraft wheel manufacturer. It is worth noting that the lateral load given in Table 1 is expressed as the ratio with the vertical load. 
Table 1 Available data for tires A and B

\begin{tabular}{ccccc}
\hline Tire & Loading case & Pressure $\mathbf{P}(\mathbf{b a r})$ & Vertical load Z $(\mathbf{d a N})$ & Lateral load ratio Y/Z \\
\hline & Inflation & 14.8 & - & - \\
$\mathrm{A}$ & Vertical & 15.5 & 22,500 & 0 \\
& Cornering & 15.5 & 22,500 & $\pm 0.23 ; \pm 0.30 ; \pm 0.53$ \\
\hline & Inflation & 14.7 & - & - \\
B & Vertical & 15.3 & 24,800 & 0 \\
& Cornering & 15.3 & 24,800 & $\pm 0.15 ; \pm 0.20 ; \pm 0.45$ \\
\hline
\end{tabular}

The distribution of tire-rim contact pressure for the different loading cases can be plotted against the curvilinear abscissa along the rim profile and the azimuth. By convention, the curvilinear abscissa is negative (resp. positive) for the inner (resp. outer) wheel half, and its origin is located, for each side, at the point of the tire-rim contact area nearest the wheel center, as shown in Fig. 10 Dashed lines are added to delimit characteristic areas of the rim profile such as the bead seat or the flange.

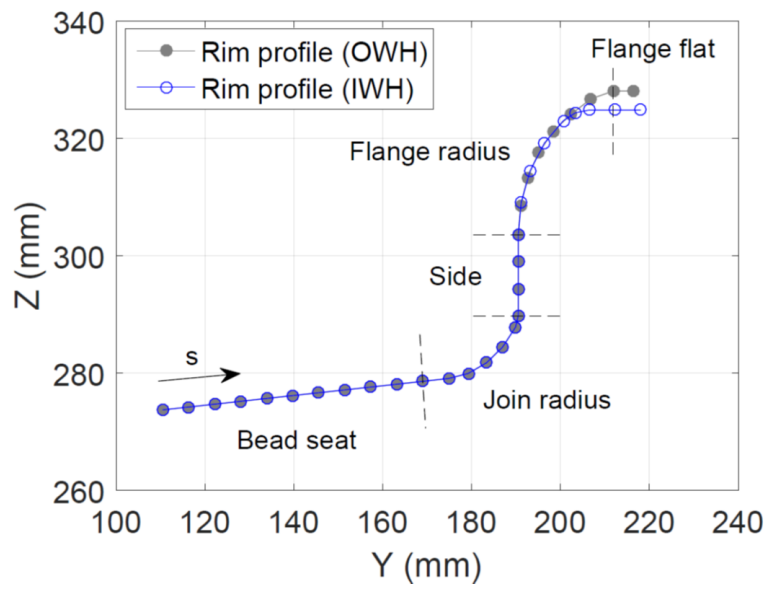

Fig. 10 Rim profile

The tire-rim contact pressure distributions given by tire manufacturers for inflation, vertical and cornering loading cases are illustrated in Figs. 11 and 12 The dashed red lines delimit the rim profile for the two wheel halves as in Fig. 10 


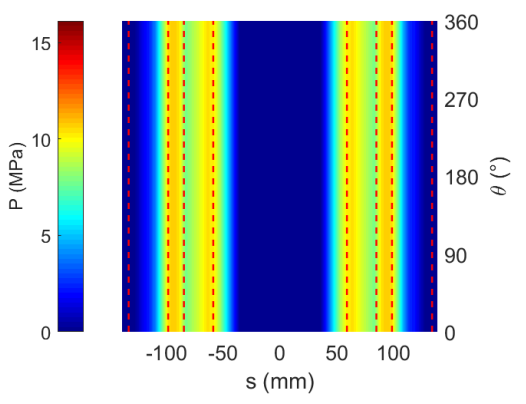

(a) Inflation case

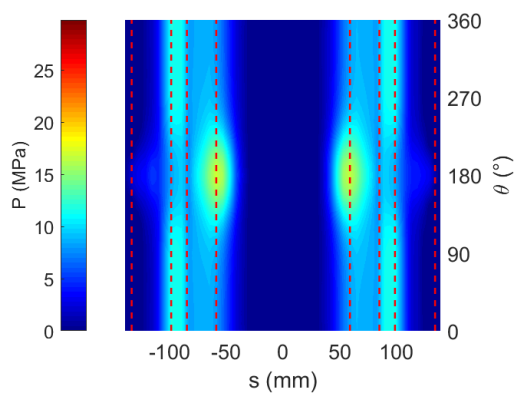

(b) Vertical loading case

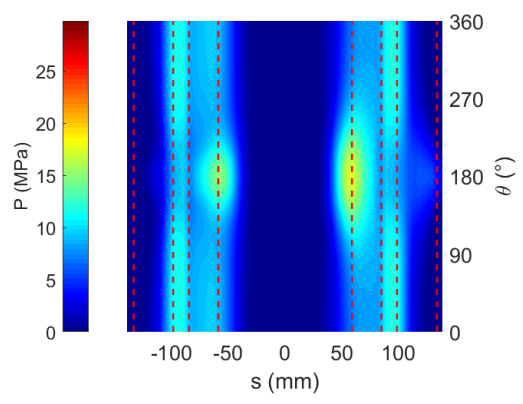

(c) Lateral loading case $(Y / Z=0.23)$

Fig. 11 Examples of tire-rim contact pressure distribution for tire A

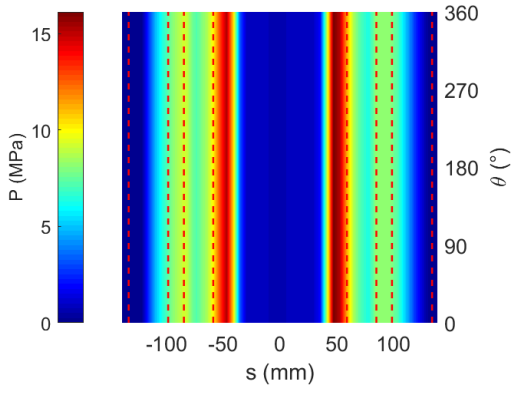

(a) Inflation

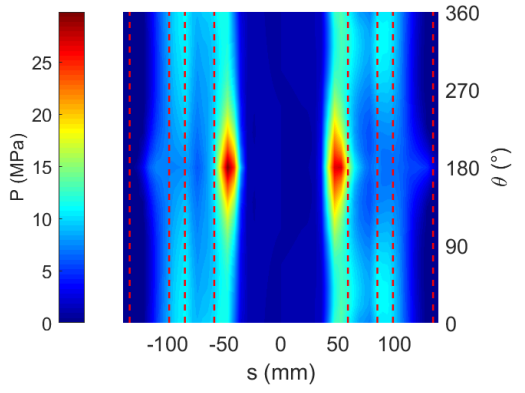

(b) Vertical loading case

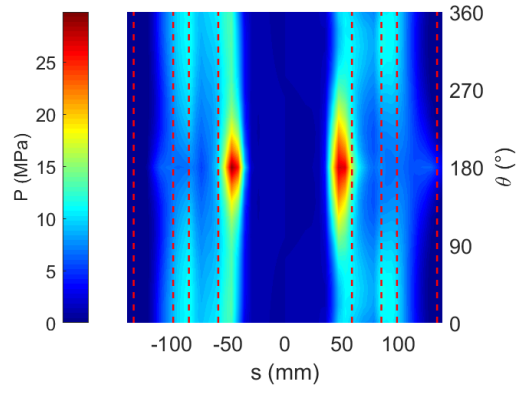

(c) Lateral loading case $(Y / Z=0.2)$

Fig. 12 Examples of tire-rim contact pressure distribution for tire $B$

Despite the fact that the studied loading cases are close, the two tires exhibit very different pressure distributions. For the inflation case, tire A shows a balanced pressure distribution between the bead seat and the flange, whereas the pressure is higher in the bead seat area for tire B. For vertical and cornering loading cases, the pressure distributions are qualitatively similar but there are still significant local differences. Moreover, the pressure amplitudes may vary by a factor of two at some points between the two tires. This variation can be partially explained by the methods used for data generation (FEA or experiments). Therefore, tire-rim interface loadings are complex and delicate to estimate. They may be very different from one tire to another, and even tire manufacturer data do not always ensure a good correlation between FEAs and experiments of aircraft wheels, making their design difficult. Thus, there is a need to precisely quantify these loadings to obtain predictive numerical simulations of the wheel structural response. In the next section, tire-rim loadings given by tire manufacturers are projected on the eigenmode basis defined in Section $\Pi$ to show whether they can be described in a common formalism. 


\section{B. Results}

\section{Tire A}

Tire-rim contact pressure distributions associated with the loading cases described in Table. 1 are converted into nodal equivalent forces and projected on the static eigenmodes of the wheel at the tire-rim interface. The elastic strain energy distribution over the modes for each loading case is obtained using Eq. (12), and illustrated in Fig. 13, where eigenmodes are ranked with increasing eigenvalue for each harmonic. Only the first 30 modes of each harmonic, among approximately 3,000, are reported in the following figures to focus on data of interest.

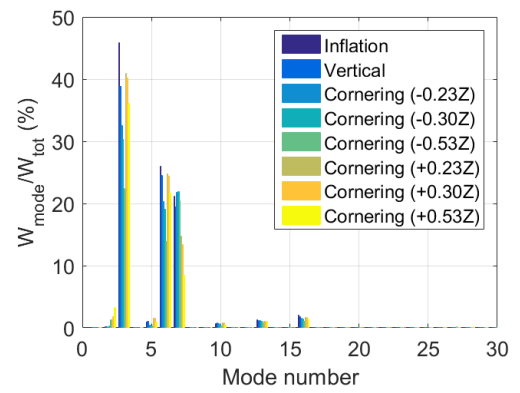

(a) Harmonic 0

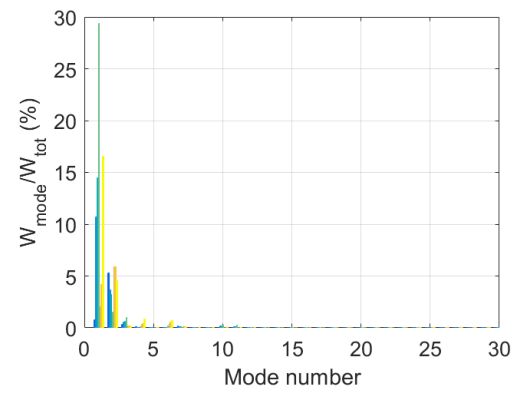

(b) Harmonic 1

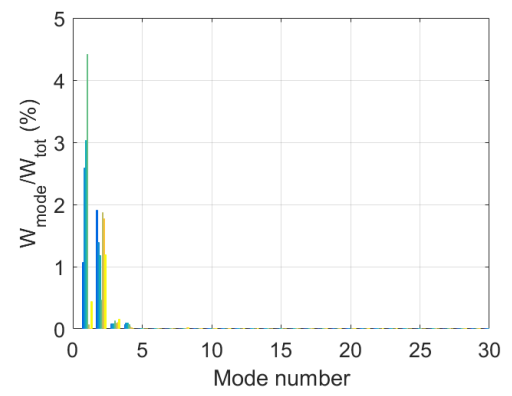

(c) Harmonic 2

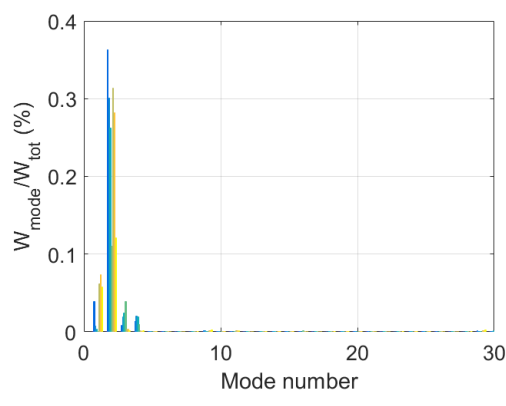

(d) Harmonic 3

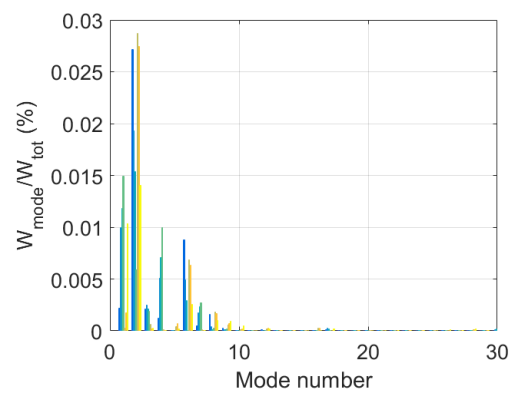

(e) Harmonic 4

Fig. 13 Elastic strain energy contribution of the tire-rim interface static eigenmodes for different loading cases

The elastic strain energy distribution is dependent on the considered loading case. The inflation case is somewhat particular because it only activates fundamental harmonic modes due to its axisymmetry. The inter-sector phase shift generated by upper harmonics is thus not necessary. For other loading cases, the excited modes in each harmonic are always the same and of low-order. Highly contributing modes belong to the first three harmonics (including the fundamental one), while third and fourth harmonic modes have a small contribution to the total elastic strain energy. Furthermore, fundamental harmonic modes are the same as for the inflation case because the tire inflation is present in all loading cases (i.e., the tire is necessarily inflated before any further loading). Contribution differences of these modes can partially be explained by the nitrogen pressure increase under loads. Therefore, although the elastic strain energy contribution of each mode is different from one loading case to an other, the dominant modes always remain the same. 
Because the dominant modes have been identified, it is necessary to assess how many of them are required to effectively approximate any tire-rim loading. Consequently, they are ranked in descending order of elastic strain energy contribution for each loading case. For example, the results for the vertical loading case are illustrated in Fig. 14. The criterion defined in Eq. (13) is then used to perform a truncation of the mode series. For $\eta=99 \%$ of the total elastic strain energy, 19 modes are required to approximate the vertical loading case. Similarly, 9 modes have to be considered for the inflation loading case and 20 for the highest cornering one. The ranking of the modes is slightly different for each loading case but a reduced set of dominant modes can be identified to encompass all of them.

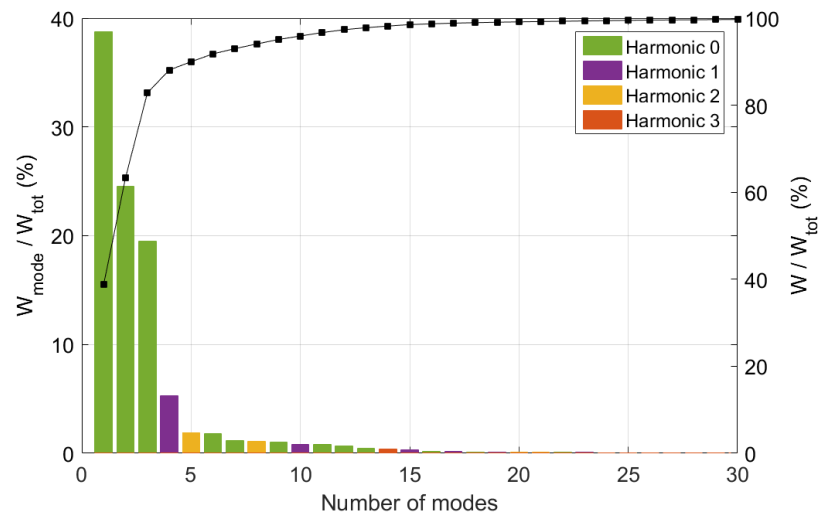

Fig. 14 Ranking in descending order of elastic strain energy contributions for the vertical loading case

However, the elastic strain energy conservation is a macroscopic criterion and it is difficult to determine the fair value of $\eta$ without other pieces of information. As proposed in Section III, resultant forces are thus investigated. The change of the resultant forces with the number of selected modes (ranked in descending order of elastic strain energy contributions) for the vertical loading case is illustrated in Fig. 15 .

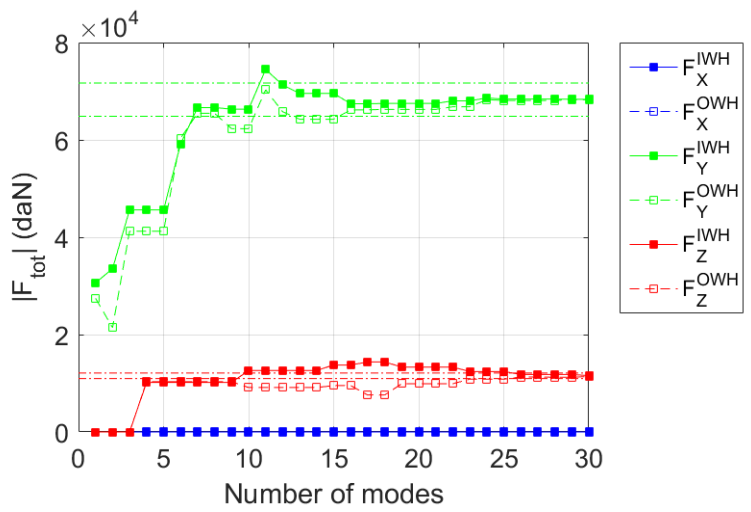

Fig. 15 Resultant forces change with the number of retained modes for the vertical loading case 
The dotted lines represent 5\% variations of the target resultant forces. Resultant forces have broadly converged with the 19 mode approximation, thereby indicating that the truncation criterion based on the total elastic strain energy conservation with $\eta=99 \%$ is a priori sufficient. The same trends are observed with the other loading cases. It is worth noting that modes of the fundamental harmonic only contribute to the axial resultant force in the $Y$-direction, whereas those of the first harmonic can only generate a vertical resultant force in the $Z$-direction, by construction. Upper harmonic modes are zero-resultant modes, which explains why the resultant forces remain constant at some points in Fig. 15. However, they can modify the tire-rim loading distribution and should not be neglected. The displacements induced by the known tire-rim loading and its approximation with 19 modes are compared in Fig. 16
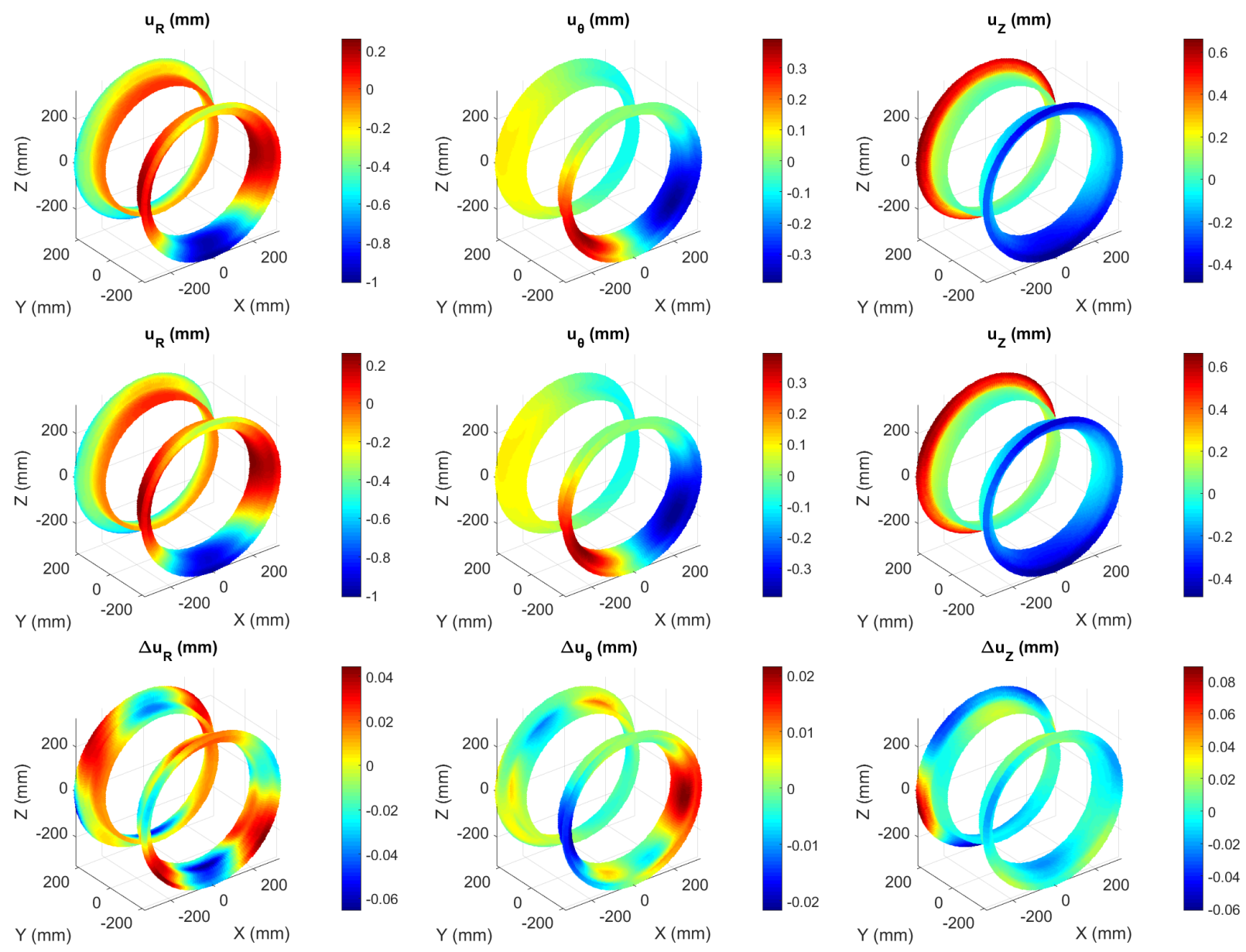

(a) Radial displacement

(b) Circumferential displacement

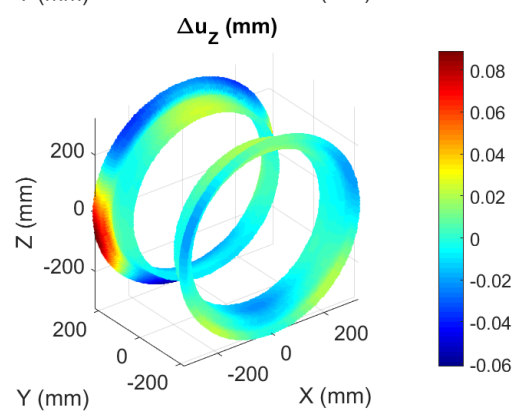

(c) Axial displacement

Fig. 16 Tire-rim interface displacements obtained with known tire-rim loading (top), 19 mode approximation (middle) and error contour (bottom) for the vertical loading case

The displacements are very close (root mean square difference, $\mathrm{RMS}=0.02 \mathrm{~mm}$ ), thereby indicating again that the truncation criterion based on the total elastic strain energy conservation with $\eta=99 \%$ is satisfactory. The structural response of the wheel should thus be well represented with this loading approximation. However, the number of 
influential modes also depends on the studied quantities of interest. This number decreases as moving away from the tire-rim interface area (i.e, Saint-Venant's principle). A correct approximation of local strains in the rim, for example, will require a higher number of modes than in the webs. Therefore, it is possible to find the right number of modes required to have a good approximation of the wheel structural response in each area. Moreover, it is worth recalling that the known tire-rim loadings used in this study are obtained considering a totally rigid wheel. It is thus questionable to absolutely try to recover the structural response due to these loadings, which can actually be different.

The results obtained with tire A show that any loading applied to the wheel by this tire can be approximated using a unique set of dominant modes. Moreover, this set is relatively small and contains approximately twenty modes. In the next section, the same work is performed for tire B.

\section{Tire $B$}

As previously, the elastic strain energy distribution over the modes for each loading case described in Table. 1 is illustrated in Fig. 17, where eigenmodes are ranked in ascending order of eigenvalue for each harmonic.

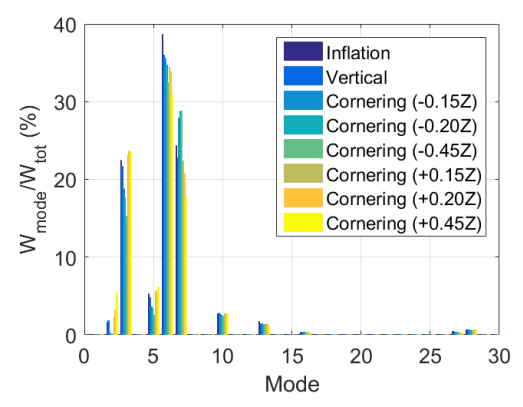

(a) Harmonic 0

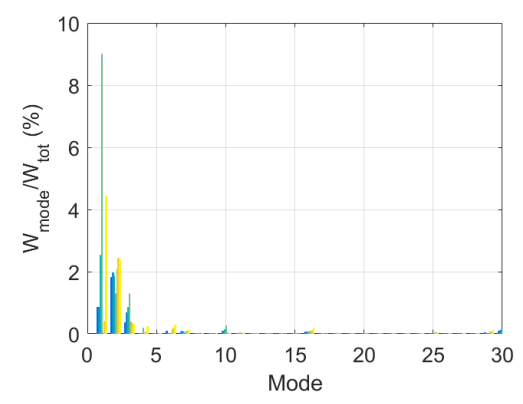

(b) Harmonic 1

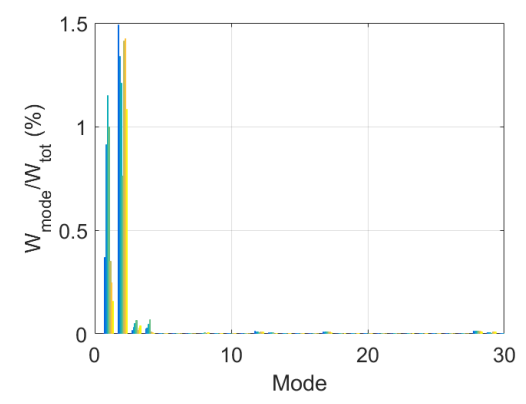

(c) Harmonic 2

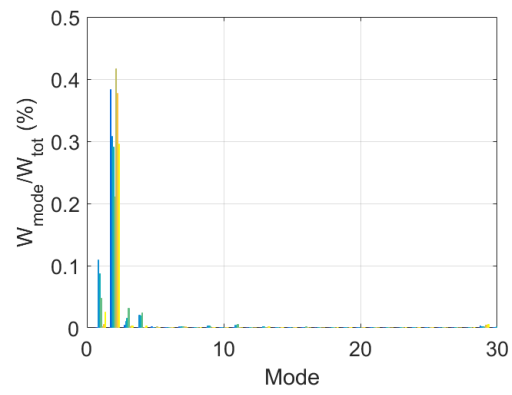

(d) Harmonic 3

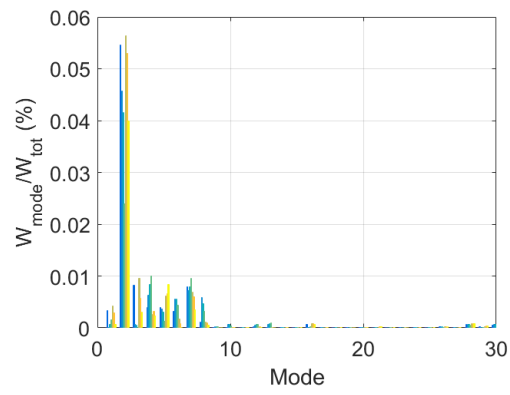

(e) Harmonic 4

Fig. 17 Elastic strain energy contribution of the tire-rim interface static eigenmodes for different loading cases

The excited modes in each harmonics are the same for all loading cases and low-order ones. Highly contributing modes belong again to the first three harmonics, and they are essentially the same as for tire A. However, the elastic strain energy distribution is less spread out. The first and second harmonic mode contributions do not exceed $10 \%$ and $1.5 \%$ respectively, while they reach up to $30 \%$ and $5 \%$ for tire A. This variation is explained by the accuracy of the tire-rim 
loading distributions (Figs. 11]12]. Data for tire B were obtained by experiments implying a poorer discretization. Once again, the modes are ranked in descending order of elastic strain energy contribution for each loading case to assess the number of modes required to effectively approximate them. The results for the vertical loading case are illustrated in Fig. 18 .

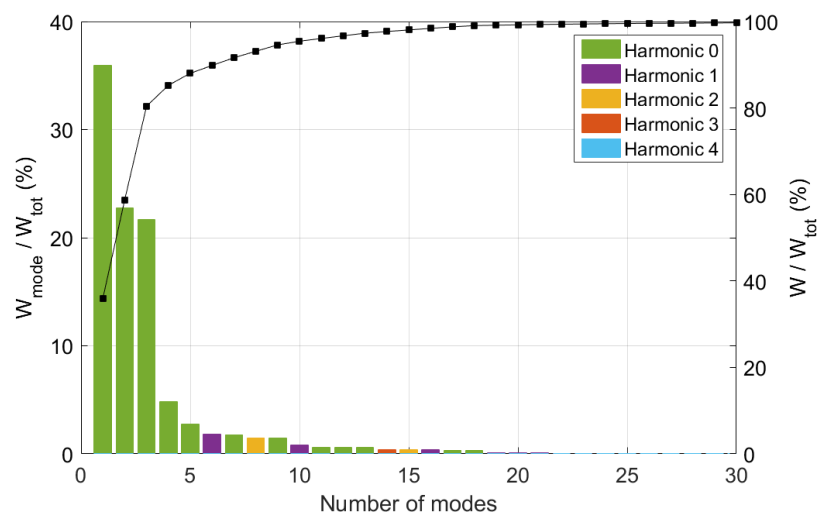

Fig. 18 Ranking in descending order of elastic strain energy contributions for the vertical loading case

For $\eta=99 \%$ of the total elastic strain energy, 18 modes are required to approximate the vertical loading case for tire $\mathrm{B}$, which is very close to the number required for tire A. The ranking in descending order of elastic strain energy contribution is not exactly the same as for tire A (Fig. 14) but among the 18 required modes, 16 are strictly identical to those of tire A. This result confirms that the loadings applied to the wheel by the two tires can be described using the same set of modes. This observation is of great interest for future inverse identifications of tire-rim loadings because the two tires can be described using the same set of modes despite their differences in behavior (Figs. 11]12). Therefore, it reasonable to assume that any loading applied to the rim could be described with this particular set of modes. Additional analyses will be requested to confirm such statement.

The change of the resultant forces with the number of retained modes (ranked in descending order of elastic strain energy contributions) for the vertical loading case is illustrated in Fig. 19 to validate the results.

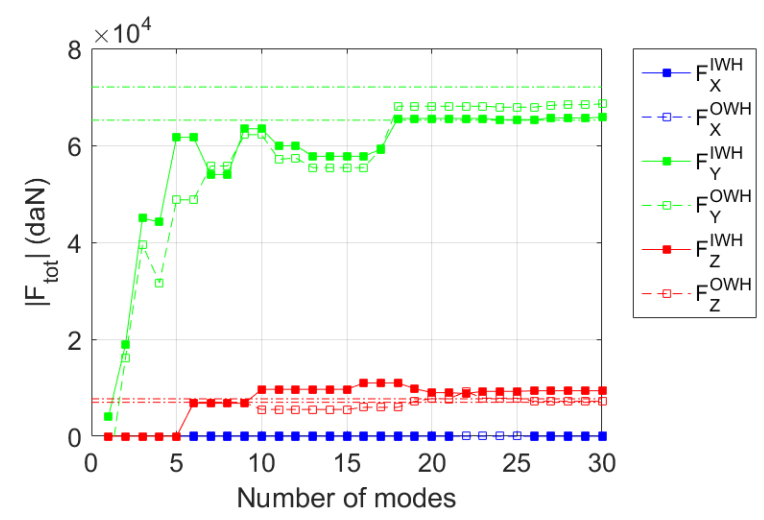

Fig. 19 Resultant forces change with the number of retained modes for the vertical loading case 
The convergence is more difficult than for tire A but the resultant forces are close to the target with the 18 mode approximation. This result demonstrates again the efficiency of the truncation criterion based on the total elastic strain energy conservation with $\eta=99 \%$.

Last, tire-rim displacements obtained with the exact tire-rim loading and its 18 mode approximation are compared in Fig. 20 The displacements are very close as with tire A $(\mathrm{RMS}=0.02 \mathrm{~mm})$. Moreover, displacements induced by the two tires are similar, whereas the tire-rim loading distributions are different (Figs.11,12). The proposed parameterization thus offers an efficient way to differentiate tire-rim loadings by comparing the excited modes and their associated amplitudes. Furthermore, it is worth recalling the efficiency of the proposed parameterization in terms of reducing the number of DOFs to describe tire-rim loadings. Table 2 reports the associated number of DOFs at the main stages of the presented framework and shows the reduction efficiency of the chosen parameterization.
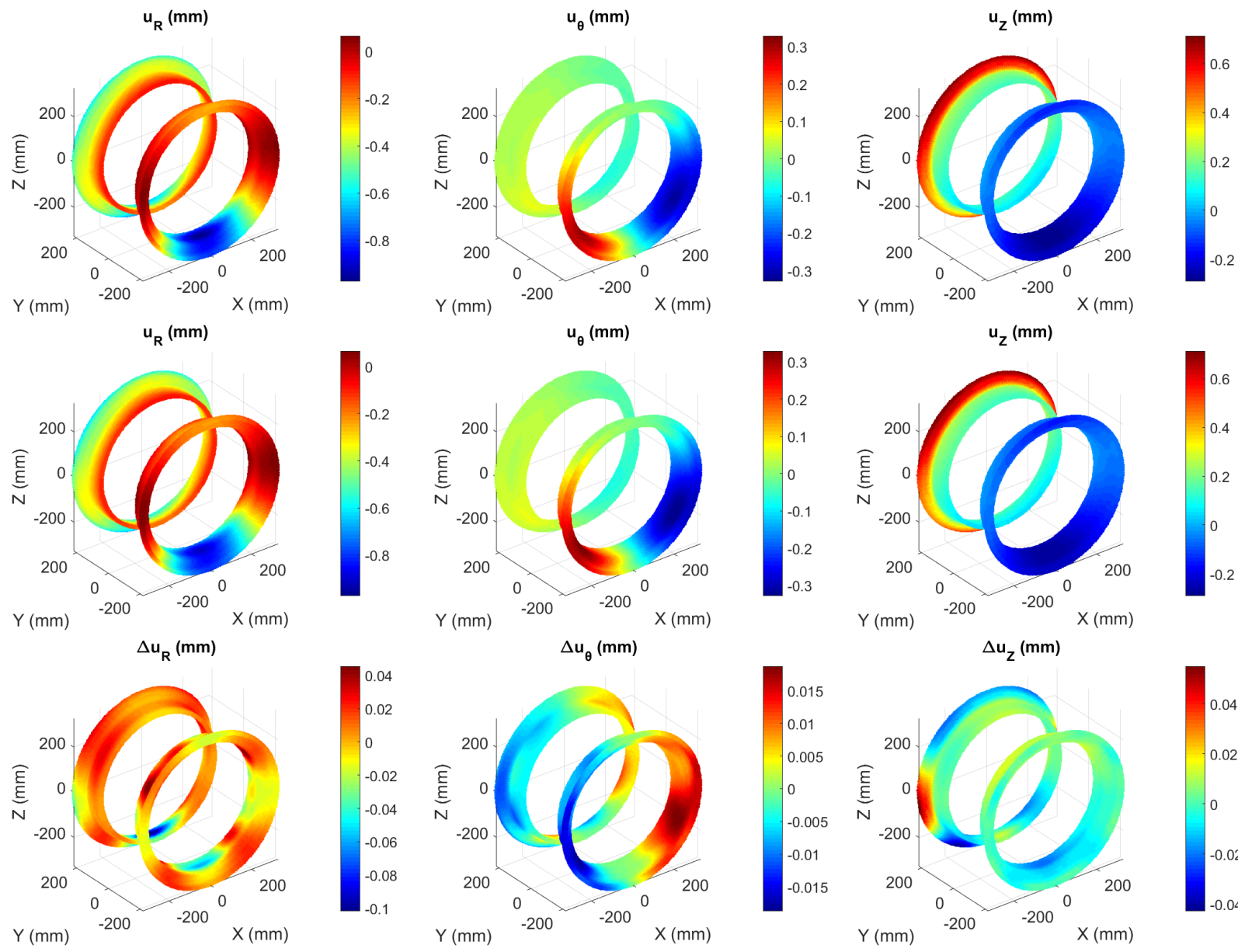

(a) Radial displacement

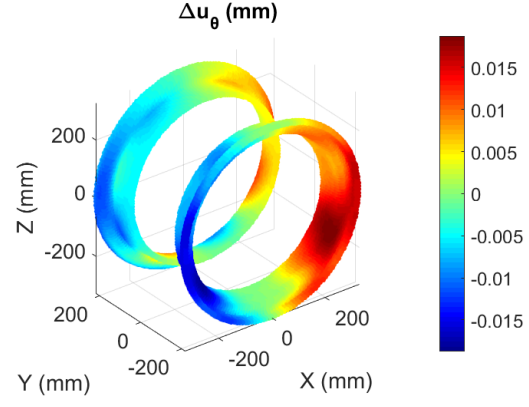

(b) Circumferential displacement

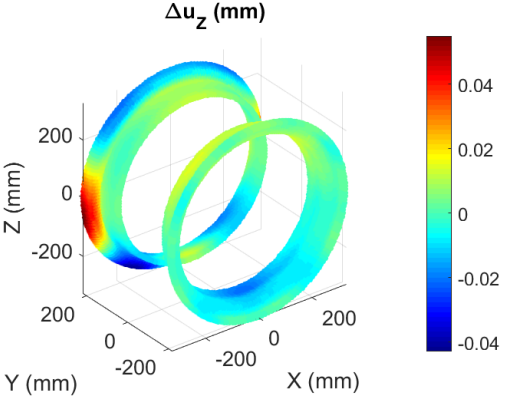

(c) Axial displacement

Fig. 20 Tire-rim interface displacements obtained with known tire-rim loading (top), 18 mode approximation (middle) and error contour (bottom) for the vertical loading case 
Table 2 Number of independent DOFs used to describe tire-rim loadings throughout the presented framework

\begin{tabular}{cc}
\hline Model & Number of DOFs \\
\hline Full linear Finite Element Model & 770,000 \\
\hline After static condensation on tire-rim interface & 30,240 \\
\hline After diagonalization and use of selection criterion & $\approx 20$ \\
\hline
\end{tabular}

\section{Conclusion}

The analysis of the tire-rim interaction for aircraft wheel design purposes has been investigated. A parameterization of the tire-rim interface loadings was proposed using model reduction techniques. The static eigenmodes of the wheel stiffness matrix that is condensed at the tire-rim interface were used to approximate any loading applied to the rim and cyclic symmetry properties proved to be useful to compute them. This parameterization is new compared to those proposed in the litterature, which are based on arbitrary shape functions or tire models, because it only relies on the aircraft wheel structure. It thus allows the tire-rim interface loadings to be ranked in a natural manner and their impact on the structural response of the wheel to be evaluated, which is very useful for the aircraft wheel manufacturer.

Moreover, a criterion based on the elastic strain energy conservation was defined to select the dominant eigenmodes required to approximate a given static loading. Projections of known tire-rim loadings, given by tire manufacturers for two different tires and several loading cases, showed that the dominant modes always remained the same and were limited in number. Therefore, the reduced basis composed of the major eigenmodes can be used to approximate any loading applied to the rim. This parameterization, which does not involve any tire modeling, and is only dependent on the wheel structure, is objective and is more compact and robust than other parameterization. It gives access to a reduced model with a minimal number of unknowns and allows very fast yet trustworthy computations. Furthermore, the proposed approach is generic and offers a convenient and efficient framework for arbitrary loadings.

Additional studies have to be performed to determine the sensitivity of critical areas of the wheel to the number of selected modes. This analysis will allow aircraft wheel manufacturers to link the required accuracy on some quantities of interest to that on tire-rim loadings. The parameterization should also be assessed on different aircraft wheel designs to identify trends. The parameterization proposed herein will then be used to perform inverse identifications of tire-rim loadings. The selection of the dominant tire-rim interface static eigenmodes based on the elastic strain energy conservation allowed those with a strong impact on the structural response of the wheel to be selected. Therefore, their amplitudes will be sought in future inverse identifications because of their potential identifiability and this will lead to new insights as to how to estimate the pressure distribution at tire-rim interfaces. 


\section{Appendix}

\section{First static condensation}

Let us consider a fundamental sector of the aircraft wheel, as shown in Fig. A1. In the following, left superscripts $l$ and $h$ denote, respectively, DOFs on the low and high boundaries of this sector, and $i$ denotes the tire-rim interface DOFs. Additional subscripts 1 and 2 distinguish DOFs of boundaries only and those belonging to boundaries and tire-rim interface simultaneously. The number of associated DOFs are designated hereafter as $n_{l}, n_{h}, n_{i}, n_{l_{1}}, n_{h_{1}}, n_{l_{2}}$, and $n_{h_{2}}$. For each node, DOFs are defined in a cylindrical coordinate system $\left(u_{R}, u_{\theta}\right.$ and $\left.u_{Z}\right)$.
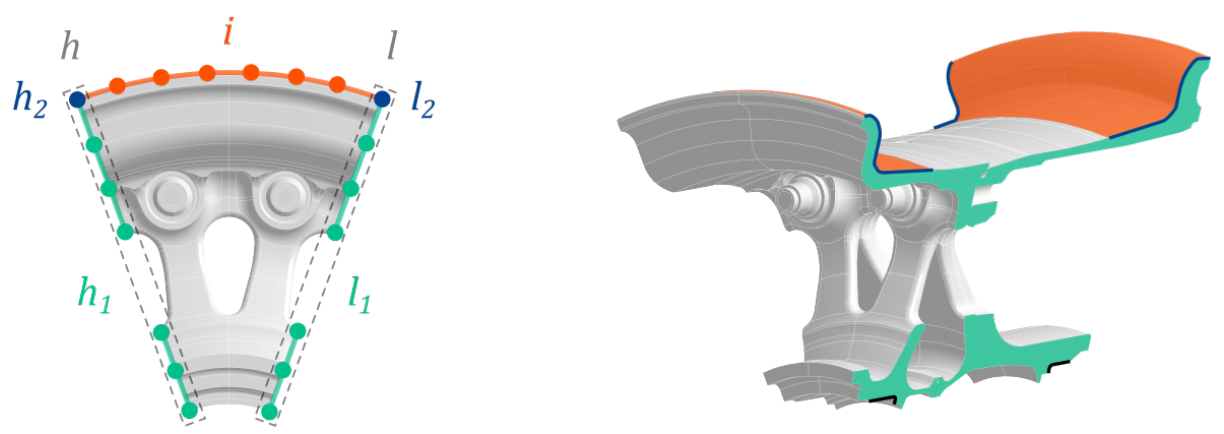

Fig. A1 Aircraft wheel fundamental sector

Static condensation of this sector on inter-sector boundaries and tire-rim interface DOFs is performed. DOFs on the bearing support surfaces are motionless. A reduced stiffness matrix is obtained

$$
\left[\widehat{\boldsymbol{K}}_{\text {sec }}^{\text {bound }+i n t}\right]=\left[\begin{array}{ccc}
{\left[{ }^{l l} \widehat{\boldsymbol{K}}\right]} & {\left[{ }^{l i} \widehat{\boldsymbol{K}}\right]} & {\left[{ }^{l h} \widehat{\boldsymbol{K}}\right]} \\
{\left[{ }^{l i} \widehat{\boldsymbol{K}}\right]^{\top}} & {\left[{ }^{i i} \widehat{\boldsymbol{K}}\right]} & {\left[{ }^{i h} \widehat{\boldsymbol{K}}\right]} \\
{\left[{ }^{l h} \widehat{\boldsymbol{K}}\right]^{\top}} & {\left[{ }^{i h} \widehat{\boldsymbol{K}}\right]^{\top}} & {\left[{ }^{h h} \widehat{\boldsymbol{K}}\right]}
\end{array}\right]
$$

with

$$
\begin{aligned}
& {\left[{ }^{l l} \widehat{\boldsymbol{K}}\right]=\left[\begin{array}{cc}
\left.{ }^{l_{1} l_{1}} \widehat{\boldsymbol{K}}\right] & {\left[{ }^{l_{1} l_{2}} \widehat{\boldsymbol{K}}\right]} \\
{\left[{ }^{{ }^{1} l_{2}} \widehat{\boldsymbol{K}}\right]^{\top}} & {\left[{ }^{l_{2} l_{2}} \widehat{\boldsymbol{K}}\right]}
\end{array}\right] \quad\left[{ }^{h h} \widehat{\boldsymbol{K}}\right]=\left[\begin{array}{ll}
\left.{ }^{h_{1} h_{1}} \widehat{\boldsymbol{K}}\right] & {\left[{ }^{h_{1} h_{2}} \widehat{\boldsymbol{K}}\right]} \\
{\left[{ }^{h_{1} h_{2}} \widehat{\boldsymbol{K}}\right]^{\top}} & {\left[{ }^{h_{2} h_{2}} \widehat{\boldsymbol{K}}\right]}
\end{array}\right] \quad\left[{ }^{l h} \widehat{\boldsymbol{K}}\right]=\left[\begin{array}{ll}
{\left[{ }^{l_{1} h_{1}} \widehat{\boldsymbol{K}}\right]} & {\left[{ }^{l_{1} h_{2}} \widehat{\boldsymbol{K}}\right]} \\
{\left[{ }^{l_{2} h_{1}} \widehat{\boldsymbol{K}}\right]} & {\left[{ }^{l_{2} h_{2}} \widehat{\boldsymbol{K}}\right]}
\end{array}\right]} \\
& {\left[{ }^{i} \widehat{\boldsymbol{K}}\right]=\left[\begin{array}{l}
{\left[{ }^{l_{1} i} \widehat{\boldsymbol{K}}\right]} \\
{\left[{ }^{l_{2} i} \widehat{\boldsymbol{K}}\right]}
\end{array}\right] \quad\left[{ }^{i h} \widehat{\boldsymbol{K}}\right]=\left[\begin{array}{ll}
{\left[{ }^{i h_{1}} \widehat{\boldsymbol{K}}\right]} & {\left[{ }^{i h_{2}} \widehat{\boldsymbol{K}}\right]}
\end{array}\right]}
\end{aligned}
$$




\section{Use of cyclic symmetry properties}

The global stiffness matrix of a structure with cyclic symmetry is block-circulant, each block being defined by the stiffness matrix of the fundamental sector [35]. By extension, the reduced stiffness matrix of the wheel at all its inter-sector boundaries and at the tire-rim interface is obtained in the same way (Fig. A2).

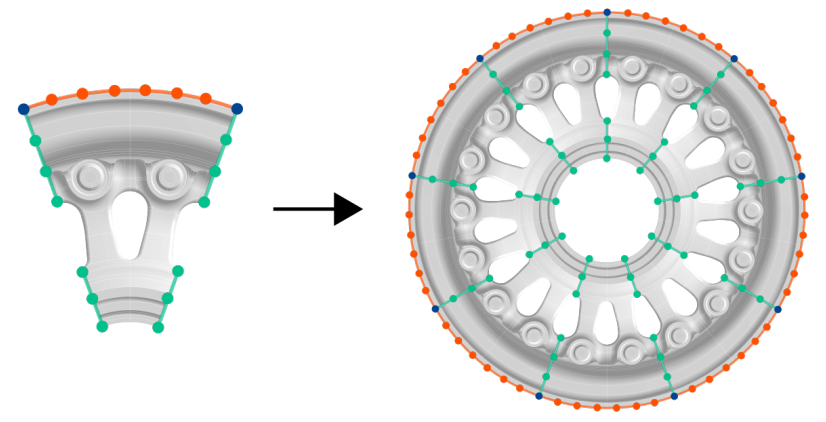

Fig. A2 Use of cyclic symmetry properties

Considering each sector as an integral part of the entire structure, the DOFs of the high boundary are viewed as those of the low boundary of the adjacent sector. Thus, the reduced DOFs for the whole structure read

$$
\left\{\widehat{\boldsymbol{u}}_{360}\right\}=\left\{\left\{\widehat{\boldsymbol{u}}_{0}\right\}\left\{\widehat{\boldsymbol{u}}_{1}\right\} \ldots\left\{\widehat{\boldsymbol{u}}_{N-1}\right\}\right\}^{\top}
$$

with

$$
\left\{\widehat{\boldsymbol{u}}_{j}\right\}=\left\{\left\{l \widehat{\boldsymbol{u}}_{j}\right\}\left\{^{i} \widehat{\boldsymbol{u}}_{j}\right\}\right\}^{\top} \quad \forall j \in[0, N-1]
$$

The reduced stiffness matrices of all sectors being equal, their assembly gives the reduced stiffness matrix of the wheel at all its inter-sector boundaries and at the tire-rim interface

$$
\left[\widehat{\boldsymbol{K}}_{360}^{\text {bound }+ \text { int }}\right]=\operatorname{Bcirc}\left(\left[\widehat{\boldsymbol{K}}_{A}\right],\left[\widehat{\boldsymbol{K}}_{B}\right],[\mathbf{0}], \ldots,[\mathbf{0}],\left[\widehat{\boldsymbol{K}}_{B}\right]^{\top}\right)
$$

with

$$
\left[\widehat{\boldsymbol{K}}_{A}\right]=\left[\begin{array}{cc}
{\left[{ }^{l l} \widehat{\boldsymbol{K}}\right]+\left[{ }^{h h} \widehat{\boldsymbol{K}}\right]} & {\left[{ }^{l i} \widehat{\boldsymbol{K}}\right]} \\
{\left[{ }^{l i} \widehat{\boldsymbol{K}}\right]} & {\left[{ }^{i i} \widehat{\boldsymbol{K}}\right]}
\end{array}\right] \quad\left[\widehat{\boldsymbol{K}}_{B}\right]=\left[\begin{array}{cc}
{\left[{ }^{l h} \widehat{\boldsymbol{K}}\right]} & {[\mathbf{0}]_{n_{l} \times n_{i}}} \\
{\left[{ }^{i h} \widehat{\boldsymbol{K}}\right]} & {[\mathbf{0}]_{n_{i} \times n_{i}}}
\end{array}\right]
$$

\section{Second static condensation}

To obtain the reduced stiffness matrix of the wheel at the tire-rim interface DOFs only, a second static condensation is required. First, the reduced stiffness matrix $\left[\widehat{\boldsymbol{K}}_{360}^{\text {bound }+i n t}\right]$ is reorganized to reveal new master and slave DOFs to be considered. Master (resp. slave) DOFs are those belonging to boundaries and tire-rim interface simultaneously (resp. 
boundaries only). They are highlighted in orange and green, respectively, in Fig. A3. The stiffness matrix becomes

$$
\left[\widehat{\boldsymbol{K}}_{360}^{\text {bound }+ \text { int }}\right]=\left[\begin{array}{ll}
{\left[\widehat{\boldsymbol{K}}_{s s}\right]} & {\left[\widehat{\boldsymbol{K}}_{s m}\right]} \\
{\left[\widehat{\boldsymbol{K}}_{m s}\right]} & {\left[\widehat{\boldsymbol{K}}_{m m}\right]}
\end{array}\right]
$$

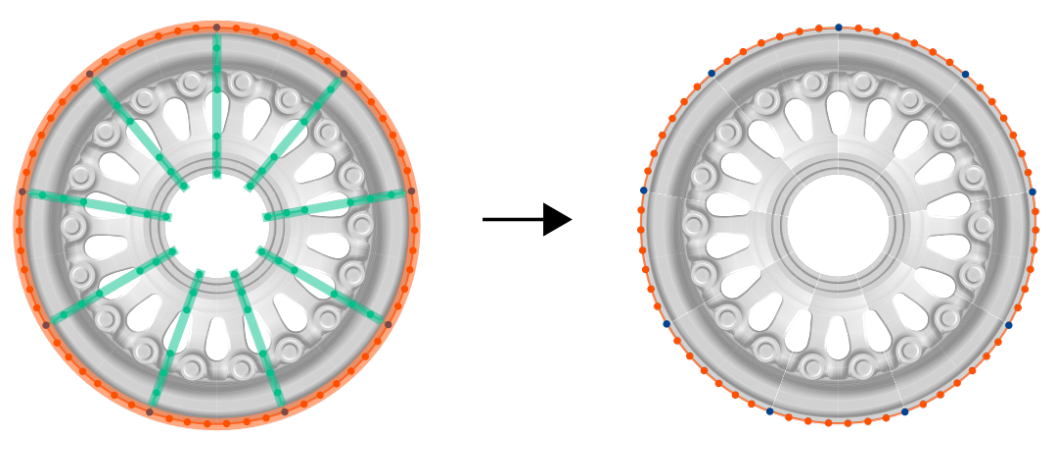

Fig. A3 Second static condensation

It is worth noting that $\left[\widehat{\boldsymbol{K}}_{s s}\right],\left[\widehat{\boldsymbol{K}}_{s m}\right],\left[\widehat{\boldsymbol{K}}_{m s}\right]$, and $\left[\widehat{\boldsymbol{K}}_{m m}\right]$ are block-circulant matrices, each block being defined by the reduced stiffness matrix of the fundamental sector. Moreover, matrices $\left[\widehat{\boldsymbol{K}}_{s s}\right]$ and $\left[\widehat{\boldsymbol{K}}_{m m}\right]$ are symmetric

$$
\begin{aligned}
& {\left[\widehat{\boldsymbol{K}}_{s s}\right]=\operatorname{Bcirc}\left(\left[{ }^{l_{1} l_{1}} \widehat{\boldsymbol{K}}\right]+\left[{ }^{h_{1} h_{1}} \widehat{\boldsymbol{K}}\right],\left[{ }^{l_{1} h_{1}} \widehat{\boldsymbol{K}}\right],[\mathbf{0}]_{n_{l_{1}} \times n_{l_{1}}}, \ldots,[\mathbf{0}]_{n_{l_{1}} \times n_{l_{1}}},\left[{ }^{l_{1} h_{1}} \widehat{\boldsymbol{K}}\right]^{\top}\right)}
\end{aligned}
$$

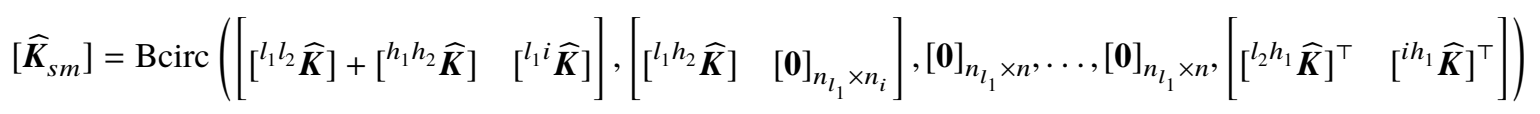

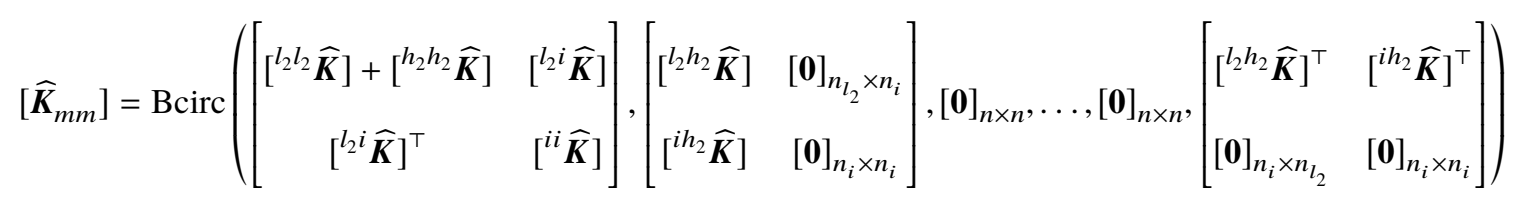

with $n=n_{l_{2}}+n_{i}$. For the sake of simplicity, the following notation is used

$$
\begin{gathered}
{\left[\widehat{\boldsymbol{K}}_{s s}\right]=\operatorname{Bcirc}\left(\left[\boldsymbol{A}_{1}\right],\left[\boldsymbol{A}_{2}\right],[\mathbf{0}]_{n_{l_{1}} \times n_{l_{1}}}, \ldots,[\mathbf{0}]_{n_{l_{1}} \times n_{l_{1}}},\left[\boldsymbol{A}_{2}\right]^{\top}\right)} \\
{\left[\widehat{\boldsymbol{K}}_{s m}\right]=\operatorname{Bcirc}\left(\left[\boldsymbol{B}_{1}\right],\left[\boldsymbol{B}_{2}\right],[\mathbf{0}]_{n_{l_{1}} \times n}, \ldots,[\mathbf{0}]_{n_{l_{1}} \times n},\left[\boldsymbol{B}_{N}\right]\right)=\left[\widehat{\boldsymbol{K}}_{m s}\right]^{\top}} \\
{\left[\widehat{\boldsymbol{K}}_{m m}\right]=\operatorname{Bcirc}\left(\left[\boldsymbol{C}_{1}\right],\left[\boldsymbol{C}_{2}\right],[\mathbf{0}]_{n \times n}, \ldots,[\mathbf{0}]_{n \times n},\left[\boldsymbol{C}_{2}\right]^{\top}\right)}
\end{gathered}
$$

Then, the reduced stiffness matrix of the wheel at the tire-rim interface DOFs is obtained using classical static condensation

$$
\left[\widehat{\boldsymbol{K}}_{360}^{i n t}\right]=\left[\widehat{\boldsymbol{K}}_{m m}\right]-\left[\widehat{\boldsymbol{K}}_{m s}\right]\left[\widehat{\boldsymbol{K}}_{s s}\right]^{-1}\left[\widehat{\boldsymbol{K}}_{s m}\right]
$$


Because the matrix $\left[\widehat{\boldsymbol{K}}_{s s}\right]$ is symmetric and block-circulant, its inverse has the same properties. Thus, because all matrices involved in Eq. A14] are block-circulant, the matrix [ $\left.\widehat{\boldsymbol{K}}_{360}^{\text {int }}\right]$ is also block-circulant. All what remains to be established is the expression of its constitutive blocks, instead of using Eq. A14 as is. The most delicate operation is to determine the terms of $\left[\widehat{\boldsymbol{K}}_{s s}\right]^{-1}$

$$
\left[\widehat{\boldsymbol{K}}_{s s}\right]^{-1}=\operatorname{Bcirc}\left(\left[\boldsymbol{D}_{1}\right],\left[\boldsymbol{D}_{2}\right], \ldots,\left[\boldsymbol{D}_{N}\right]\right)
$$

Vescovo [36] proposed a simple and short procedure to compute the inverse of a block-circulant matrix based on the discrete Fourier transform. The constitutive blocks of $\left[\widehat{\boldsymbol{K}}_{s s}\right]^{-1}$ are expressed as

$$
\left[\boldsymbol{D}_{p}\right]=\mathfrak{R}\left(\frac{1}{N^{2}} \sum_{q=1}^{N} \exp \left(\frac{2 \pi i(q-1)(p-1)}{N}\right) \times\left[A^{(q)}\right]^{-1}\right) \quad \forall p \in[1, N]
$$

with

$$
\left[\boldsymbol{A}^{(q)}\right]=\frac{1}{N} \sum_{p=1}^{N}\left[\boldsymbol{A}_{p}\right] \exp \left(\frac{-2 \pi i(q-1)(p-1)}{N}\right) \quad \forall q \in[1, N]
$$

Next, matrice products of Eq. A14 are computed

$$
\left[\widehat{\boldsymbol{K}}_{m s}\right]\left[\widehat{\boldsymbol{K}}_{s s}\right]^{-1}=\operatorname{Bcirc}\left(\left[\boldsymbol{E}_{1}\right],\left[\boldsymbol{E}_{2}\right], \ldots,\left[\boldsymbol{E}_{N}\right]\right)
$$

and

$$
\left[\widehat{\boldsymbol{K}}_{m s}\right]\left[\widehat{\boldsymbol{K}}_{s s}\right]^{-1}\left[\widehat{\boldsymbol{K}}_{s m}\right]=\operatorname{Bcirc}\left(\left[\boldsymbol{F}_{1}\right],\left[\boldsymbol{F}_{2}\right], \ldots,\left[\boldsymbol{F}_{N}\right]\right)
$$

with

$$
\begin{gathered}
{\left[\boldsymbol{E}_{p}\right]=\left[\boldsymbol{B}_{1}\right]^{\top}\left[\boldsymbol{D}_{p}\right]+\left[\boldsymbol{B}_{2}\right]^{\top} d_{1}\left(\left[\boldsymbol{D}_{p}\right]\right)+\left[\boldsymbol{B}_{N}\right]^{\top} d_{N-1}\left(\left[\boldsymbol{D}_{p}\right]\right) \quad \forall p \in[1, N]} \\
{\left[\boldsymbol{F}_{p}\right]=\left[\boldsymbol{E}_{p}\right]\left[\boldsymbol{B}_{1}\right]+d_{N-1}\left(\left[\boldsymbol{E}_{p}\right]\right)\left[\boldsymbol{B}_{2}\right]+d_{1}\left(\left[\boldsymbol{E}_{p}\right]\right)\left[\boldsymbol{B}_{N}\right] \quad \forall p \in[1, N]}
\end{gathered}
$$

and

$$
d_{k}\left(\left[\boldsymbol{D}_{p}\right]\right)=\left\{\begin{array}{ccc}
{\left[\boldsymbol{D}_{p+k}\right]} & \text { if } & p+k \leq N \\
{\left[\boldsymbol{D}_{p+k-N}\right]} & \text { if } & p+k>N
\end{array}\right.
$$

Last, the expression of each block of the reduced stiffness matrix of the wheel at tire-rim interface DOFs is obtained

$$
\left[\widehat{\boldsymbol{K}}_{360}^{i n t}\right]=\operatorname{Bcirc}\left(\left[\boldsymbol{G}_{1}\right],\left[\boldsymbol{G}_{2}\right], \ldots,\left[\boldsymbol{G}_{N}\right]\right)
$$

with

$$
\left[\boldsymbol{G}_{p}\right]=\left[\boldsymbol{C}_{p}\right]-\left[\boldsymbol{F}_{p}\right] \quad \forall p \in[1, N]
$$


Therefore, it is not necessary to build the matrix in its entirety, thereby requiring less storage capacity. Moreover, it can be noted that this matrix is symmetric

$$
\left[\boldsymbol{G}_{p}\right]=\left[\boldsymbol{G}_{N-p+2}\right]^{\top} \quad \forall p \in[L, N]
$$

with $L=(N+1) / 2$ if $N$ is odd and $L=N / 2+1$ if $N$ is even.

\section{Diagonalization}

Because the reduced stiffness matrix of the wheel at the tire-rim interface is known, it may be diagonalized to obtain the tire-rim interface static eigenmodes

$$
\left(\left[\widehat{\boldsymbol{K}}_{360}^{i n t}\right]-\lambda_{i}[\boldsymbol{I}]\right)\left\{\boldsymbol{\Phi}_{i}\right\}=\{\boldsymbol{0}\}
$$

However, the diagonalization can be performed in a more efficient way than solving the previous equation directly. According to the general theory of the linear representation of finite groups [37, 38] (and following the works of Fortescue [39], MacNeal et al. [40], and Thomas [41]), the quantity $\left\{\widehat{\boldsymbol{u}}_{j}\right\}$ is expressed in physical coordinates for the $j$ th sector as a finite sum of harmonic functions

$$
\left\{\widehat{\boldsymbol{u}}_{j}\right\}=\left\{\widetilde{\boldsymbol{u}}_{0}^{0}\right\}+\sum_{k=1}^{K}\left[\left\{\tilde{\boldsymbol{u}}_{0}^{k, c}\right\} \cos (j k \alpha)+\left\{\tilde{\boldsymbol{u}}_{0}^{k, s}\right\} \sin (j k \alpha)\right]+(-1)^{j}\left\{\widetilde{\boldsymbol{u}}_{0}^{N / 2}\right\}
$$

with $K=(N-1) / 2$ if $N$ is odd, and $K=N / 2-1$ if $N$ is even, and where $\left\{\widetilde{\boldsymbol{u}}_{0}^{k}\right\}$ are the complex harmonic components of order $k$, also called cyclic components, defined on the fundamental sector (generally associated with subscript 0 ). By ordering physical and cyclic components of the entire structure such that

$$
\begin{aligned}
& \{\widehat{\boldsymbol{u}}\}=\left\{\begin{array}{llll}
\left\{\widehat{\boldsymbol{u}}_{0}\right\} & \left\{\widehat{\boldsymbol{u}}_{1}\right\} & \ldots & \left\{\widehat{\boldsymbol{u}}_{N-1}\right\}
\end{array}\right\}^{\top}
\end{aligned}
$$

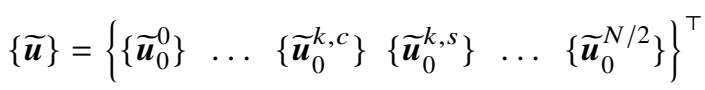

Equation A27) is written in a matrix form

$$
\{\widehat{\boldsymbol{u}}\}=([\mathcal{F}] \otimes[\boldsymbol{I}])\{\widetilde{\boldsymbol{u}}\}
$$

where $\otimes$ is the Kronecker product, $[\boldsymbol{I}]$ the identity matrix of size $\left(n_{l_{2}}+n_{i}\right) \times\left(n_{l_{2}}+n_{i}\right)$, and $[\mathcal{F}]$ the real-valued Fourier matrix. Using the above coordinate transformation equation, the expression of the reduced stiffness matrix $\left[\widehat{\boldsymbol{K}}_{360}^{\text {int }}\right]$ is obtained in the cyclic basis

$$
[\widetilde{\boldsymbol{K}}]=\left([\mathcal{F}]^{\top} \otimes[\boldsymbol{I}]\right)\left[\widehat{\boldsymbol{K}}_{360}^{\text {int }}\right]([\mathcal{F}] \otimes[\boldsymbol{I}])
$$


Because $\left[\widehat{\boldsymbol{K}}_{360}^{i n t}\right]$ is block-circulant, the transformation in cyclic components gives a pseudo-block-diagonal matrix $[\widetilde{\boldsymbol{K}}]$ where each block $\left[\widetilde{\boldsymbol{K}}^{k}\right]$ is defined by

$$
\begin{gathered}
{\left[\widetilde{\boldsymbol{K}}^{0}\right]=\sum_{p=1}^{N}\left[\boldsymbol{G}_{p}\right]} \\
{\left[\widetilde{\boldsymbol{K}}^{k}\right]=\left[\begin{array}{cc}
\sum_{p=1}^{N}\left[\boldsymbol{G}_{p}\right] \cos (k \alpha(p-1)) & \sum_{p=1}^{N}\left[\boldsymbol{G}_{p}\right] \sin (k \alpha(p-1)) \\
-\sum_{p=1}^{N}\left[\boldsymbol{G}_{p}\right] \sin (k \alpha(p-1)) & \sum_{p=1}^{N}\left[\boldsymbol{G}_{p}\right] \cos (k \alpha(p-1))
\end{array}\right]} \\
{\left[\widetilde{\boldsymbol{K}}^{N / 2}\right]=\sum_{p=1}^{N}(-1)^{p-1}\left[\boldsymbol{G}_{p}\right]}
\end{gathered}
$$

Thus, the physical eigenproblem from Eq. [A26] is split into several smaller sub-problems defined in the cyclic basis on the fundamental sector of the wheel and depending upon the harmonic index $k$

$$
\left[\widetilde{\boldsymbol{K}}^{k}\right]\left\{\widetilde{\boldsymbol{\Phi}}_{0}^{k}\right\}=\lambda\left\{\widetilde{\boldsymbol{\Phi}}_{0}^{k}\right\}
$$

Solutions to these eigenproblems are classified into two categories [38]. The first one corresponds to non-degenerated solutions for which $\left[\widetilde{\boldsymbol{K}}^{k}\right]$ is an $\left(n_{l_{2}}+n_{i}\right) \times\left(n_{l_{2}}+n_{i}\right)$ matrix, and the multiplicity of the eigenvalue $\lambda$ is one. These solutions are associated with harmonic index $k=0$ and $k=N / 2$ if $N$ is even. For $k=0$, all sectors are in phase, while two adjacent sectors are out of phase for $k=N / 2$. The second category corresponds to degenerated solutions for which $\left[\widetilde{\boldsymbol{K}}^{k}\right]$ is a $2\left(n_{l_{2}}+n_{i}\right) \times 2\left(n_{l_{2}}+n_{i}\right)$ matrix, and the multiplicity of the eigenvalue $\lambda$ is two. These solutions are associated with harmonic indices $k \in[1, K]$. The two eigenmodes associated with an eigenvalue are orthogonal and any linear combination is also a valid eigenmode. Once the eigenproblems have been solved in the cyclic basis, the eigenmodes of the entire structure are retrieved in the physical basis applying Eq. A27) for each sector $j \in[0, N-1]$. They are reconstructed separately for each harmonic index $k$

$$
\begin{gathered}
\{\boldsymbol{\Phi}\}=\left(\left\{\mathcal{F}^{0}\right\} \otimes[\boldsymbol{I}]\right)\left\{\widetilde{\boldsymbol{\Phi}}_{0}^{0}\right\} \quad \text { for } \quad k=0 \\
\{\boldsymbol{\Phi}\}=\left(\left[\mathcal{F}^{k}\right] \otimes[\boldsymbol{I}]\right)\left[\begin{array}{l}
\left\{\widetilde{\boldsymbol{\Phi}}_{0}^{k, c}\right\} \\
\left\{\widetilde{\boldsymbol{\Phi}}_{0}^{k, s}\right\}
\end{array}\right] \quad \forall k \in[1, K] \\
\{\boldsymbol{\Phi}\}=\left(\left\{\mathcal{F}^{N / 2}\right\} \otimes[\boldsymbol{I}]\right)\left\{\widetilde{\boldsymbol{\Phi}}_{0}^{N / 2}\right\} \quad \text { for } \quad k=N / 2
\end{gathered}
$$


with

$$
\left\{\mathcal{F}^{0}\right\}=\frac{1}{\sqrt{N}}\left\{\begin{array}{c}
1 \\
1 \\
1 \\
\vdots \\
1
\end{array}\right\} \quad ; \quad\left[\mathcal{F}^{k}\right]=\sqrt{\frac{2}{N}}\left[\begin{array}{cc}
1 & 0 \\
\vdots & \vdots \\
\cos (j k \alpha) & \sin (j k \alpha) \\
\vdots & \vdots \\
\cos ((N-1) j k \alpha) & \sin ((N-1) j k \alpha)
\end{array}\right] \quad ; \quad\left\{\mathcal{F}^{N / 2}\right\}=\frac{1}{\sqrt{N}}\left\{\begin{array}{c}
1 \\
-1 \\
1 \\
\vdots \\
-1
\end{array}\right\}
$$

For degenerated solutions, nodal diameters are observed in the mode shapes corresponding to lines of zero displacement in the structure.

The use of the cyclic symmetry allows the modal analysis to be carried out through the sole manipulation of the fundamental sector, which represents a huge gain in terms of computation time and storage, without any compromise on the quality of the result.

\section{Funding Sources}

This work was funded by Safran Landing Systems and ANRT. It is part of a doctoral project under Industrial Convention of Formation by Research (CIFRE).

\section{Acknowledgments}

The authors acknowledge useful discussions with Jean-Frédéric Diebold, Methods \& Tools Team Leader, and Prof. René Billardon, Senior Expert in Mechanics, from Safran Landing Systems site at Vélizy-Villacoublay, France.

\section{References}

[1] Nakagiri, S., and Suzuki, K., "Finite element interval analysis of external loads identified by displacement input with uncertainty," Computer Methods in Applied Mechanics and Engineering, Vol. 168, No. 1-4, 1999, pp. 63-72. doi:10.1016/S00457825(98)00134-0.

[2] Turco, E., "A boundary element approach to identify static boundary conditions in elastic solids from stresses at internal points," Inverse Problems in Engineering, Vol. 7, No. 4, 1999, pp. 309-333. doi:10.1080/174159799088027700.

[3] Chock, J. M. K., and Kapania, R. K., "Load updating for finite element models," AIAA Journal, Vol. 41, No. 9, 2003, pp. 1667-1673. doi:10.2514/2.7312.

[4] Turco, E., "Is the statistical approach suitable for identifying actions on structures?" Computers \& Structures, Vol. 83, No. 25-26, 2005, pp. 2112-2120. doi:10.1016/j.compstruc.2005.03.006. 
[5] Amiot, F., Hild, F., and Roger, J. P., "Identification of elastic property and loading fields from full-field displacement measurements," International Journal of Solids and Structures, Vol. 44, No. 9, 2007, pp. 2863-2887. doi:10.1016/j.ijsolstr. 2006.08.031

[6] Xu, S., Deng, X., Tiwari, V., Sutton, M. A., Fourney, W. L., and Bretall, D., “An inverse approach for pressure load identification,” International Journal of Impact Engineering, Vol. 37, No. 7, 2010, pp. 865-877. doi:10.1016/j.ijimpeng.2009.10.007.

[7] Martini, D., Hochard, C., and Charles, J. P., "Load identification for full-field reconstruction: applications to plates under tension loads," International Journal for Numerical Methods in Engineering, Vol. 91, No. 10, 2012, pp. $1073-1086$. doi:10.1002/nme.4304.

[8] Nakamura, T., Igawa, H., and Kanda, A., "Inverse identification of continuously distributed loads using strain data," Aerospace Science and Technology, Vol. 23, No. 1, 2012, pp. 75-84. doi:10.1016/j.ast.2011.06.012.

[9] Technical standard order (TSO) C135a - Transport airplane wheels and wheel and brake assemblies, Federal Aviation Administration, Washington, District of Columbia, 2008.

[10] Europen technical standard order (ETSO) C135a - Large aeroplane wheels and wheel and brake assemblies, European Aviation Safety Agency, Köln, Germany, 2010.

[11] Zaharov, S. P., "Feasibility study of how to make compression measurements of the tire bead against the rim," Kauchuk $i$ Rezina, Vol. 16, No. 8, 1957, pp. 30-31.

[12] Balabin, I. V., and Zubarev, N. A., "Force interaction between a wide profile tire and the wheel rim," Automobil'naya Promyshlennost, Vol. 30, No. 6, 1964, pp. 16-19.

[13] Walter, J. D., and Kiminecz, R. K., "Bead contact pressure measurements at the tire-rim interface," SAE Technical Paper 750458, 1975. doi:10.4271/750458.

[14] Sherwood, J. A., Fussell, B. K., Edwards, W. R., Gross, T. S., and Watt, D. W., "Study of the pressure distribution on an aircraft tire-wheel interface,” Journal of Aircraft, Vol. 32, No. 5, 1995, pp. 921-928. doi:10.2514/3.46819.

[15] Wan, X., Shan, Y., Liu, X., He, T., and Wang, J., “Tire-rim interface pressure of a commercial vehicle wheel under radial loads: theory and experiment," Measurement Science and Technology, Vol. 28, No. 11, 2017, pp. 1-9. doi:10.1088/1361-6501/aa8895.

[16] Sherwood, J. A., Ayres, J. M., Gross, T. S., and Watt, D. W., "An investigation of tire-wheel interface loads using ADINA," Computers and structures, Vol. 56, No. 2-3, 1995, pp. 377-387. doi:10.1016/0045-7949(95)00030-K.

[17] Spencer, B. F., Kirkner, D. J., Schudt, E. E., and Kandarpa, S., “Aircraft wheel life assessment,” Tech. rep., University of Notre Dame, 1993.

[18] Kirkner, D. J., Spencer, B. F., Schudt, E. E., Kandarpa, S., and Chawla, M. D., "Determination of tire-wheel interface pressure distribution for aircraft wheels," 34th Structures, Structural Dynamics and Materials Conference, AIAA, 1993, pp. 415-423. doi:10.2514/6.1993-1343. 
[19] Schudt, E. E., Kirkner, D. J., Spencer, B. F., and Chawla, M., "Three-dimensional verification of an axisymmetric algorithm for tire-wheel interface load recovery,” SAE Technical Paper 941176, 1994. doi:10.4271/941176.

[20] Kandarpa, S., Spencer, B. F., Kirkner, D. J., and Champion, M., "Determination of tire-wheel interface loads for aircraft wheels," Journal of Aircraft, Vol. 31, No. 2, 1994, pp. 433-440. doi:10.2514/3.46504.

[21] Chen, C. H. S., "Prediction of bead area contact load at the tire-wheel interface using NASTRAN," Tenth NASTRAN Users' Colloquium, NASA, Washington, District of Columbia, 1982, pp. 133-142.

[22] Tseng, N. T., Pelle, R. G., and Chang, J. P., "Numerical simulation for the tire-rim interface for a radial passenger tire,” $S A E$ Technical Paper 890641, 1989. doi:10.4271/890641.

[23] Meng, J., Ping, Z., Ji, Q., and Liu, Z., “An improved pressure distribution model of automotive wheel's radial fatigue,” Applied Mechanics and Materials, Vol. 201-202, 2012, pp. 287-291. doi:10.4028/www.scientific.net/AMM.201-202.287.

[24] Ballo, F., Previati, G., Gobbi, M., and Mastinu, G., "A semi-analytical tyre model for the study of tyre/rim interaction on a road vehicle," ASME 2017 International Design Engineering Technical Conferences and Computers and Information in Engineering Conference, ASME, 2017. doi:10.1115/DETC2017-67730.

[25] Gras, R., Mellé, D., and Hild, F., "Loading identification on a tire/rim contact for an inflation pressure," Proceedings of the BSSM 10th International Conference on Advances in Experimental Mechanics, 2015.

[26] Cosseron, K., Gras, R., Mellé, D., Diebold, J.-F., Hild, F., and Roux, S., "Inverse identification of the loading applied by a tire on a landing gear wheel," Residual Stress, Thermomechanics \& Infrared Imaging, Hybrid Techniques and Inverse Problems, Conference Proceedings of the Society for Experimental Mechanics Series, Vol. 7, Springer, Cham, 2019 , pp. 13-16. doi:10.1007/978-3-319-95074-7_3.

[27] Stearns, J., Srivatsan, T. S., Gao, X., and Lam, P. C., "Understanding the influence of pressure and radial loads on stress and displacement response of a rotating body: the automobile wheel," International Journal of Rotating Machinery, Vol. 2006, 2006, pp. 1-8. doi:10.1155/IJRM/2006/60193.

[28] 2018 Aircraft year book, Tire and Rim Association, Inc., Copley, Ohio, 2018.

[29] Guyan, R. J., "Reduction of stiffness and mass matrices," AIAA Journal, Vol. 3, No. 2, 1965, pp. 380-380. doi:10.2514/3.2874.

[30] Sobieszczanski-Sobieski, J., and Hajela, P., "Accuracy of an approximate static structural analysis technique based on stiffness matrix eigenmodes," 20th Structures, Structural Dynamics, and Materials Conference, NASA, 1979, pp. 127-136. doi:10.2514/6.1979-748.

[31] ANSYS mechanical APDL substructuring analysis guide, ANSYS, Inc., Canonsburg, Pennsylvania, 2017.

[32] Weyl, H., Symmetry, Princeton University Press, Princeton, New Jersey, 1952. 
[33] Bajan, R. L., Feng, C. C., and Jaszlics, I. J., "Vibration analysis of complex structural systems by modal substitution," Shock and Vibration Bulletin, Vol. 39, No. 3, 1969, pp. 99-105.

[34] Balmès, E., "Optimal Ritz vectors for component mode synthesis using the singular value decomposition," AIAA Journal, Vol. 34, No. 6, 1996, pp. 1256-1260. doi:10.2514/3.13221.

[35] Bladh, R., "Efficient predictions of the vibratory response of mistuned bladed disks by reduced order modeling," Ph.D. thesis, Mechanics [physics.med-ph], University of Michigan, 2001.

[36] Vescovo, R., "Inversion of block-circulant matrices and circular array approach," IEEE Transactions on Antennas and Propagation, Vol. 45, No. 10, 1997, pp. 1565-1567. doi:10.1109/8.633869.

[37] Valid, R., and Ohayon, R., "Static and dynamic analysis of cyclically symmetric structures," La Recherche Aérospatiale, Vol. 4, 1985, pp. 41-53.

[38] Ohayon, R., and Soize, C., Structural acoustics and vibration: mechanical models, variational formulations and discretization, Academic Press, San Diego, California, 1998, Chap. 3.

[39] Fortescue, C. L., "Method of symmetrical co-ordinates applied to the solution of polyphase networks," Transactions of the American Institute of Electrical Engineers, Vol. 37, No. 2, 1918, pp. 1027-1140. doi:10.1109/T-AIEE.1918.4765570.

[40] MacNeal, R. H., Harder, R. L., and Mason, J. B., “NASTRAN cyclic symmetry capability,” NASTRAN: Users' experiences, NASA, Washington, District of Columbia, 1973, pp. 395-421.

[41] Thomas, D. L., "Dynamics of rotationally periodic structures," International Journal for Numerical Methods in Engineering, Vol. 14, No. 1, 1979, pp. 81-102. doi:10.1002/nme.1620140107. 